\title{
Parameterization of basal friction near grounding lines in a one-dimensional ice sheet model
}

\author{
G. R. Leguy ${ }^{1,2}$, X. S. Asay-Davis ${ }^{2,3,4}$, and W. H. Lipscomb ${ }^{2}$ \\ ${ }^{1}$ New Mexico Institute of Mining and Technology, 801 Leroy Place, Socorro, \\ New Mexico 87501, USA \\ ${ }^{2}$ Los Alamos National Laboratory, Los Alamos, New Mexico 87545, USA \\ ${ }^{3}$ Courant Institute of Mathematical Sciences, New York University, 251 Mercer Street, \\ New York, New York 10012-1185, USA \\ ${ }^{4}$ Potsdam Institute for Climate Impact Research, Telegraphenberg A 31, 14473 Potsdam, Germany
}

Correspondence to: G. R. Leguy (gunter@nmt.edu)

Received: 4 December 2013 - Published in The Cryosphere Discuss.: 14 January 2014

Revised: 16 May 2014 - Accepted: 5 June 2014 - Published: 18 July 2014

\begin{abstract}
Ice sheets and ice shelves are linked by the transition zone, the region where flow dominated by vertical shear stress makes a transition to flow dominated by extensional stress. Adequate resolution of the transition zone is necessary for numerically accurate ice sheet-ice shelf simulations. The required resolution depends on how the basal physics is parameterized. We propose a new, simple parameterization of the effective pressure near the grounding line, combined with an existing friction law linking effective pressure to basal stress and sliding, in a one-dimensional, fixed-grid, vertically integrated model. This parameterization represents connectivity between the basal hydrological system and the ocean in the transition zone. Our model produces a smooth transition between finite basal friction in the ice sheet and zero basal friction in the ice shelf. In a set of experiments based on the Marine Ice Sheet Model Intercomparison Project (MISMIP), we show that with a smoother basal shear stress, the model yields accurate steady-state results at a fixed-grid resolution of $\sim 1 \mathrm{~km}$.
\end{abstract}

\section{Introduction}

Antarctica's contribution to sea level rise has increased in the past decade. While the contribution of the East Antarctic Ice Sheet (EAIS) remains steady, mass loss from the West Antarctic Ice Sheet (WAIS) has more than doubled (Velicogna, 2009; Rignot et al., 2011). Theoretical models suggest that marine ice sheets like WAIS are susceptible to instabilities when they lie on bedrock that slopes upward in the direction of ice flow (Weertman, 1974; Schoof, 2007a). If these instabilities are triggered, mass loss will accelerate, exacerbating future sea-level rise and potentially leading to WAIS collapse (Vaughan and Spouge, 2002; Joughin and Alley, 2011). For this reason it is important to understand the dynamic processes that drive ice sheets in the region.

The glaciology community has developed many ice-sheet models of varying complexity. Stokes models (Durand et al., 2009; Favier et al., 2012), which include all components of the stress tensor, are the most accurate of the widely used iceflow models. However, they can be impractical at continental scales because of their large computational cost, especially in three dimensions. By neglecting terms in the stress tensor, modelers have derived and applied several simpler, computationally cheaper approximations to the Stokes equations, including the first-order model (Pattyn, 2003; Perego et al., 2012), the so-called L1L2 model (Hindmarsh, 2004; Schoof and Hindmarsh, 2010; Cornford et al., 2013), the shallow-ice approximation (Rutt et al., 2009), the shallow-shelf approximation (MacAyeal, 1989; Schoof, 2007a), and hybrid models that combine the shallow-ice and shallow-shelf approximations (Bueler and Brown, 2009; Pollard and DeConto, 2012). Shallow-ice models do not include extensional stresses and therefore cannot accurately represent shelf flow. Even the more accurate flow approximations require very fine resolution $(<1 \mathrm{~km})$ in the transition zone (the region where 
ice-sheet flow dominated by vertical shear stress transitions to ice-shelf flow dominated by extensional stress) in order to obtain numerically accurate ice sheet-ice shelf simulations (Durand et al., 2009; Cornford et al., 2013).

Several studies have investigated the effects of different friction laws on ice dynamics using one-dimensional, depth-integrated models (Muszynski and Birchfield, 1987; MacAyeal, 1989; Schoof, 2007a). Vieli and Payne (2005) and Schoof (2007a) prescribed a discontinuous friction law across the grounding line where the ice loses contact with the bed. In these models the friction is nonzero in the ice sheet, but abruptly falls to zero at the grounding line. These models have the drawback that very high grid resolution near the grounding line is required for convergence. In models with fixed grids, a tolerance of a few kilometers in the grounding-line location requires a resolution on the order of tens to hundreds of meters (Durand et al., 2009; Gladstone et al., 2010a, b; Cornford et al., 2013), which is computationally prohibitive for large-scale simulations. This requirement was confirmed by the Marine Ice Sheet Model Intercomparison Project (MISMIP, Pattyn et al., 2012) which used the same basal friction law as in Schoof (2007a). In this project, participants using a variety of fixed-grid models found that the errors in grounding-line position were unacceptably high $(100 \mathrm{~km}$ or more) at resolutions that were computationally feasible in three-dimensional models $(\sim 1 \mathrm{~km})$.

One way to reduce the computational cost is to use adaptive mesh refinement (Goldberg et al., 2009; Gladstone et al., 2010b; Cornford et al., 2013), i.e., to subdivide the horizontal mesh near features where high resolution is needed. Durand et al. (2009) investigated this approach in a Stokes model with the basal friction law of Schoof (2007a). They performed a set of experiments based on the MISMIP experiments with the goal of reaching steady state when using very high resolution near the grounding line. Even with grid resolution of $30 \mathrm{~m}$ in the transition zone, they found differences in the grounding-line position over an advance-and-retreat cycle of $\sim 2 \mathrm{~km}$, whereas theoretical arguments predict that there should be no difference.

In order to reduce the need for high resolution near the grounding line, Pattyn et al. (2006) proposed a smooth basalfriction parameter that decays exponentially to zero as the ice flows across the grounding line into the ice shelf. This approach gave promising results, as the transition zone could be partially resolved even at $12.5 \mathrm{~km}$ grid resolution. However, the model introduced an arbitrary length scale of exponential decay, and the basal friction remained nonzero (though small) in the ice shelf. Gladstone et al. (2012) showed that the need for high resolution could also be relaxed by decreasing the value of the basal drag coefficient, the ice softness, channel width (when buttressing is included in the model), or by steepening the slope of the bedrock topography. Pattyn et al. (2006) and Gladstone et al. (2010a) also showed that higherorder interpolation at the grounding line, where the grounded ice sheet meets the floating ice shelf, could greatly reduce the error in the grounding-line position, implying convergence at coarser resolution.

In the case of rapidly sliding ice streams, basal resistance is controlled by the underlying water-laden plastic till (Tulaczyk et al., 2000a, b; van der Wel et al., 2013). The presence of liquid water lowers the effective pressure at the ice base, leading to reduced basal friction (Tulaczyk et al., 2000b; Carter and Fricker, 2012; van der Wel et al., 2013), an effect not accounted for in many ice sheet models. Recent observations confirm the existence of basal drainage channels that connect subglacial lake systems (Wingham et al., 2006; Fricker et al., 2009). Some of these drainage systems are found near grounding lines (Fricker and Scambos, 2009; Carter and Fricker, 2012), meaning that they are likely to connect to the ocean (Le Brocq et al., 2013). In a detailed hydrology/till model, van der Wel et al. (2013) found that subglacial conduits can extend to the grounding line if sufficient water is available from local melting and upstream transport. They concluded that the Kamb Ice Stream currently does not have conduit systems but that the Rutford Ice Stream is connected to the ocean via a permanent conduit system. Cuffey and Paterson (2010, p. 283) suggested that a free connection between subglacial water and the ocean is likely near the grounding line, though not plausible at 50 or $100 \mathrm{~km}$ upstream.

Several previous models have included the effect of basal water pressure or meltwater depth in their friction laws. Bueler and Brown (2009) assumed plastic flow with a yield stress proportional to the effective pressure $N$ (the difference between the ice overburden pressure and the basal water pressure). They parameterized basal water pressure as a linear function of water depth, with a maximum value equal to $95 \%$ of overburden pressure. Pimentel et al. (2010) used the friction law of Schoof (2005), which predicts a basal shear stress proportional to $N$ in the limit of fast flow and small $N$. They treated basal water pressure as a nonlinear function of water depth, capped at the overburden pressure. Martin et al. (2011) assumed plastic flow with a yield stress proportional to $N$, with basal water pressure prescribed to be $96 \%$ of overburden pressure under the marine portion of the Antarctic Ice Sheet (including close to grounding lines). This parameterization reduced but did not eliminate the discontinuity in basal friction at the grounding line.

The earlier models of Budd et al. (1979) and Budd and Jenssen (1989) included the effect of hydrological connectivity between basal channels and the ocean. These models assumed that the basal water pressure is equal to the ocean pressure at the same depth, or equivalently, that the effective pressure is proportional to the thickness above flotation. This implies $N=0$ at the grounding line, where the ice begins to float. Although Schoof (2005) later showed that the friction law in Budd et al. (1979) and Budd and Jenssen (1989) was unphysical, the parameterization of $N$ as a function of thickness above flotation inspired our own study. 
In this paper we propose a new treatment of effective pressure near the grounding line, combined with an established friction law (Schoof, 2005) linking basal stress and sliding to the effective pressure. In Sect. 2 we present our onedimensional, vertically integrated flowline model, including the new parameterization. We also discuss mathematical limits of the basal friction law and the numerical methods used for our simulations. In Sect. 3 we show simulation results with different values of the effective-pressure parameter $p$ for different bedrock topographies. In Sect. 4 we discuss the limitations of the model and the implications for future development of three-dimensional ice-sheet models. Sect. 5 summarizes the main results. A more detailed description of the numerical method is provided in Appendix C.

\section{Model}

The shallow-shelf flowline model presented in this paper, which is similar to the model of Schoof (2007a), is onedimensional, symmetric and depth-integrated. It is intended to represent the motion of a transversely and vertically averaged ice stream. It includes the effect of three stress terms: the longitudinal stress $\left(\tau_{1}\right)$, the basal stress $\left(\tau_{\mathrm{b}}\right)$, and the driving stress $\left(\tau_{\mathrm{d}}\right)$. The model neglects lateral shear (and therefore buttressing) and vertical shear, and thus is best used to simulate fast-flowing ice streams. While additional physics would be required to model realistic ice sheets, our model is a simple, computationally efficient tool for idealized studies of grounding-line dynamics.

\subsection{Model equations}

The model consists of an equation for the evolution of ice thickness (conservation of mass) and a vertically integrated stress-balance equation:

$H_{\mathrm{t}}+(u H)_{x}=a$,

$\left(H \tau_{1}\right)_{x}-\tau_{\mathrm{b}}+\tau_{\mathrm{d}}=0$,

where subscripts $x$ and $t$ denote partial derivatives (e.g., $\left.H_{\mathrm{t}} \equiv \frac{\partial H}{\partial t}\right)$. The ice thickness $H$, ice velocity $u$, and other important model variables are defined in Table 1. Table 2 gives the value of the accumulation rate $a$ and other model parameters. The longitudinal stress $\tau_{1}$ is vertically averaged, so that $H \tau_{1}$ is the vertically integrated stress. Derivations of Eqs. (1) and (2) can be found in Muszynski and Birchfield (1987) and MacAyeal (1989). From Schoof (2007a), the longitudinaland driving-stress terms are

$$
\begin{aligned}
& \left(H \tau_{1}\right)_{x}=\left[2 \bar{A}^{-\frac{1}{n}} H\left|u_{x}\right|^{\frac{1}{n}-1} u_{x}\right]_{x}, \\
& \tau_{\mathrm{d}}=-\rho_{\mathrm{i}} g H s_{x} .
\end{aligned}
$$

In Eq. (3), the stress $\tau_{1}$ includes the nonlinear viscosity given by Glen's flow law, where $\bar{A}$ is the depth-averaged ice softness and $n$ is the Glen's flow exponent. In Eq. (4), $\tau_{\mathrm{d}}$ is the
Table 1. Model variables.

\begin{tabular}{cll}
\hline Variables & Units & Definition \\
\hline$H$ & $\mathrm{~km}^{-1}$ & Ice thickness \\
$u$ & $\mathrm{~m} \mathrm{a}^{-1}$ & Ice velocity \\
$b$ & $\mathrm{~km}$ & Ice sheet bed elevation, positive downward \\
$s$ & $\mathrm{~km}$ & Surface elevation \\
$x_{\mathrm{g}}$ & $\mathrm{km}$ & Horizontal grounding line position \\
\hline
\end{tabular}

gravitational stress that drives ice flow in the direction of decreasing surface elevation, where $\rho_{\mathrm{i}}, g$ and $s_{x}$ are ice density, gravitational acceleration and ice surface slope, respectively.

Equations (1)-(4) apply to both the ice sheet and the ice shelf. The surface elevation $s$ is computed differently in the two regions - from the bedrock elevation and ice thickness in the ice sheet, and from exact flotation in the ice shelf:

$s=\left\{\begin{array}{cc}H-b & x<x_{\mathrm{g}} \\ \left(1-\frac{\rho_{\mathrm{i}}}{\rho_{\mathrm{w}}}\right) H & x \geq x_{\mathrm{g}}\end{array}\right.$,

where $b$ is the bedrock elevation and $\rho_{w}$ is the seawater density. We adopt the convention of Schoof (2007a) that $b$ is positive below sea level.

Basal stress beneath ice shelves is zero everywhere. Under the ice sheet, the basal-friction law takes the form given in Schoof (2005):

$\tau_{\mathrm{b}}=C|u|^{\frac{1}{n}-1} u\left(\frac{N^{n}}{\frac{m_{\max }}{\lambda_{\max } A_{\mathrm{b}}}|u|+N^{n}}\right)^{\frac{1}{n}}$,

where $C$ is the constant shear stress factor defined in Schoof (2007a), the effective pressure $N \equiv p_{\mathrm{i}}-p_{\mathrm{w}}$ is the difference between the overburden pressure $p_{\mathrm{i}} \equiv \rho_{\mathrm{i}} g H$ and the basal water pressure $p_{\mathrm{w}}, A_{\mathrm{b}}$ is the ice softness at the bed chosen based on an ice temperature of $-2^{\circ} \mathrm{C}$, and $\lambda_{\max }$ and $m_{\max }$ are the wavelength of bedrock bumps and the maximum bed obstacle slope, respectively. These last two parameters represent bedrock roughness at scales too small to be resolved in the model. As we will discuss further in Sect. 2.2, Eq. (6) was proposed in Schoof (2005) as an ad hoc nonlinear extension of the linear friction law $(n=1)$ with the appropriate behavior in the limits of both slow-flowing, thick ice in the ice-sheet interior and more rapidly sliding, thinner ice near grounding lines. Gagliardini et al. (2007) numerically validated this ad hoc formulation as a limiting case of their own friction law. We have modified the notation from Schoof (2005) to match that of Schoof (2007a) in the limit of slow flow and large effective pressure.

We assume the ice sheet to be symmetric at the ice divide, the origin of the domain, leading to the following boundary conditions:

$$
\begin{array}{lll}
u=0 & \text { at } & x=0, \\
(H-b)_{x}=0 & \text { at } & x=0,
\end{array}
$$


Table 2. Parameter values used for all experiments.

\begin{tabular}{lrll}
\hline Parameters & Value & Units & Definition \\
\hline$\rho_{\mathrm{i}}$ & 900 & $\mathrm{~kg} \mathrm{~m}^{-3}$ & Ice density \\
$\rho_{\mathrm{W}}$ & 1000 & $\mathrm{~kg} \mathrm{~m}^{-3}$ & Water density \\
$A$ & see Table 3 and Sect. 3.2 & $\mathrm{~Pa}^{-3} \mathrm{~s}^{-1}$ & Ice softness \\
$A_{\mathrm{b}}$ & $3.1688 \times 10^{-24}$ & $\mathrm{~Pa}^{-3} \mathrm{~s}^{-1}$ & Ice softness at the bed \\
$C$ & $7.62 \times 10^{6}$ & $\left.\mathrm{~Pa}^{-1} \mathrm{~s}\right)^{1 / 3}$ & Shear stress factor \\
$a$ & 0.3 & $\mathrm{~m} \mathrm{a}^{-1}$ & Ice accumulation rate \\
$g$ & 9.8 & $\mathrm{~m} \mathrm{~s}^{-2}$ & Gravitational acceleration \\
$n$ & 3 & - & Glen's flaw low exponent \\
$m_{\max }$ & 0.5 & - & Maximum bed obstacle slope \\
$\lambda_{\max }$ & 2 & $\mathrm{~m}$ & Wavelength of bedrock bumps \\
\hline
\end{tabular}

At the grounding line, the requirement of exact flotation leads to the boundary condition

$H=\frac{\rho_{\mathrm{w}}}{\rho_{\mathrm{i}}} b \quad$ at $\quad x=x_{\mathrm{g}}$.

Combining Eqs. (2)-(5), the stress balance in the ice shelf is given by

$$
\left[2 \bar{A}^{-\frac{1}{n}} H\left|u_{x}\right|^{\frac{1}{n}-1} u_{x}\right]_{x}-\rho_{\mathrm{i}}\left(1-\frac{\rho_{\mathrm{i}}}{\rho_{\mathrm{w}}}\right) g H H_{x}=0 .
$$

At the calving front the ice shelf is subject to the ocean back pressure, $p_{\mathrm{w}}=-\rho_{\mathrm{w}} g z$, between the ice shelf base, $z=\left(\rho_{\mathrm{i}} / \rho_{\mathrm{w}}\right) H$, and sea level, $z=0$. The ocean pressure partially (but not completely) balances the hydrostatic pressure of the ice, $p_{\mathrm{i}}=-\rho_{\mathrm{i}} g(z-s)$. The force on the ice shelf due to the difference in hydrostatic pressure between the ice shelf and the ocean is

$$
\begin{aligned}
f_{\mathrm{p}}\left(x_{\mathrm{c}}\right) & =\int_{-\left(\rho_{\mathrm{i}} / \rho_{\mathrm{w}}\right) H}^{s}-\rho_{\mathrm{i}} g(z-s) d z-\int_{-\left(\rho_{\mathrm{i}} / \rho_{\mathrm{w}}\right) H}^{0}-\rho_{\mathrm{w}} g z d z \\
& =\frac{1}{2} \rho_{\mathrm{i}}\left(1-\frac{\rho_{\mathrm{i}}}{\rho_{\mathrm{w}}}\right) g H^{2} .
\end{aligned}
$$

The force on the calving face due to longitudinal (viscous) stress must compensate for this imbalance in hydrostatic pressure:

$2 \bar{A}^{-\frac{1}{n}} H\left|u_{x}\right|^{\frac{1}{n}-1} u_{x}=\frac{1}{2} \rho_{\mathrm{i}}\left(1-\frac{\rho_{\mathrm{i}}}{\rho_{\mathrm{w}}}\right) g H^{2}$ at $x=x_{\mathrm{c}}$.

Following Schoof (2007a), we integrate Eq. (10) from the calving front $\left(x=x_{\mathrm{c}}\right)$ to the grounding line $\left(x=x_{\mathrm{g}}\right)$, and use Eq. (12) to show that the same condition holds at the grounding line as at the calving front:

$2 \bar{A}^{-\frac{1}{n}} H\left|u_{x}\right|^{\frac{1}{n}-1} u_{x}=\frac{1}{2} \rho_{\mathrm{i}}\left(1-\frac{\rho_{\mathrm{i}}}{\rho_{\mathrm{W}}}\right) g H^{2} \quad$ at $x=x_{\mathrm{g}}$.

In order for the stresses to remain finite, $H, u$ and $u_{x}$ must be continuous across the grounding line.

\subsection{Effective pressure parameterization and friction law}

Most models of marine ice sheets assume that the basal friction jumps discontinuously to zero across the grounding line. We propose a simple parameterization that removes the discontinuity, yielding a smooth transition between grounded and floating ice. We adopt the friction law from Schoof (2005), validated and extended in Gagliardini et al. (2007). This formulation, given by Eq. (6), has the correct limits for large values of the effective basal pressure $N$ and slow flow, and reduces to Coulomb friction in the limit of small $N$ and fast flow. Schoof (2005) suggested that this friction law is an appropriate simplification for rough terrain; Gagliardini et al. (2007) showed that this limiting case of their more general friction law (corresponding to their decay parameter $q=1$ ) was appropriate for sawtooth terrain. They also argued that this limit of their friction law may lead to better behavior in numerical models because the relation between basal stress and sliding velocity is monotonic.

If the effective pressure is continuous across the grounding line, the basal shear stress smoothly approaches zero at the grounding line. Assuming that the subglacial drainage system is connected to the ocean, the water pressure at the icesheet base will be close to the ocean pressure at that depth, reaching the ocean pressure at the grounding line (with pressure differences driving flow through the drainage system). A simple function for the effective pressure that accounts for connectivity between the subglacial drainage system and the ocean is

$N(p)=\rho_{\mathrm{i}} g H\left(1-\frac{H_{\mathrm{f}}}{H}\right)^{p}$,

in which we introduce a parameter $p$ that varies between zero (no basal water pressure) and one (the subglacial drainage system is hydrologically well connected to the ocean). The flotation thickness is defined by $H_{\mathrm{f}} \equiv \max \left(0, \frac{\rho_{\mathrm{w}}}{\rho_{\mathrm{i}}} b\right)$. The effective basal pressure $N(p)$ has the following desired limits: 
- When $p=0, N(p)=\rho_{\mathrm{i}} g H$ (no water-pressure support).

- When $p=1, N(p)=\rho_{\mathrm{i}} g\left(H-H_{\mathrm{f}}\right)$ (full water-pressure support from the ocean wherever the ice-sheet base is below sea level).

- At the grounding line when $p>0, N(p)=0$ ( $\tau_{\mathrm{b}}$ is continuous across the grounding line).

- Far from the grounding line (where the bed is above sea level and $\left.H_{\mathrm{f}}=0\right), N(p)=\rho_{\mathrm{i}} g H$.

When $H_{\mathrm{f}} / H \ll 1$ and the bedrock is below sea level $(b>0)$, the basal water pressure is attenuated to a fraction $p$ of the full ocean pressure at the depth of the bed (see Appendix A). These conditions will typically hold on the inland side of the transition zone, since a rapid increase in $H$ is usually needed to produce the driving stress that balances the relatively large basal friction in this region. One way such attenuation might occur is by a gradual loss of connectivity between the basal hydrological system and the ocean.

Equation (14) can be regarded as a mathematical regularization, ensuring that the basal friction transitions smoothly from a finite value in the ice sheet interior to zero in the ice shelf. It can also be viewed as a simple parameterization of basal hydrology, motivated by the hydrological connectivity that may exist between the ice bed and the ocean near the grounding line. (By "parameterization" we mean the replacement of small-scale or complex physical processes with a simplified process.) The functional form of Eq. (14) is ad hoc, since there are no detailed observations to show how $N$ varies near grounding lines, but the limits are physically based.

We emphasize that Eq. (14) does not represent all the processes that might be included in a complex hydrology or till model. It represents only the portion of water-pressure support related to the ocean; basal water pressure in the model falls to zero when the bedrock reaches sea level $(b=0)$. More sophisticated models of basal till find that the basal water pressure remains a significant fraction of the overburden pressure in much of the ice-sheet interior (Tulaczyk et al., 2000b; van der Wel et al., 2013). A more complex model might include a network of channels as well as water-laden till at the base of ice streams. This hydrological network would influence the basal friction through water-pressure support outside the transition zone. Thus, our parameterization predicts a larger $N$ away from the grounding line than would likely be observed in much of the interior of ice sheets. We do not think this is a critical model weakness, however, because we are mainly interested in ice dynamics near the grounding line. In the interior, where $N$ is larger, the basal shear stress is described by a power law (see Eq. (16) below) and is relatively insensitive to $N$.

Figures 1 and 2 show typical ice-sheet geometry, thickness, $H_{\mathrm{f}} / H$ and $N$ for five values of $p$ over linear and polynomial bedrock topography, respectively. In both cases, the
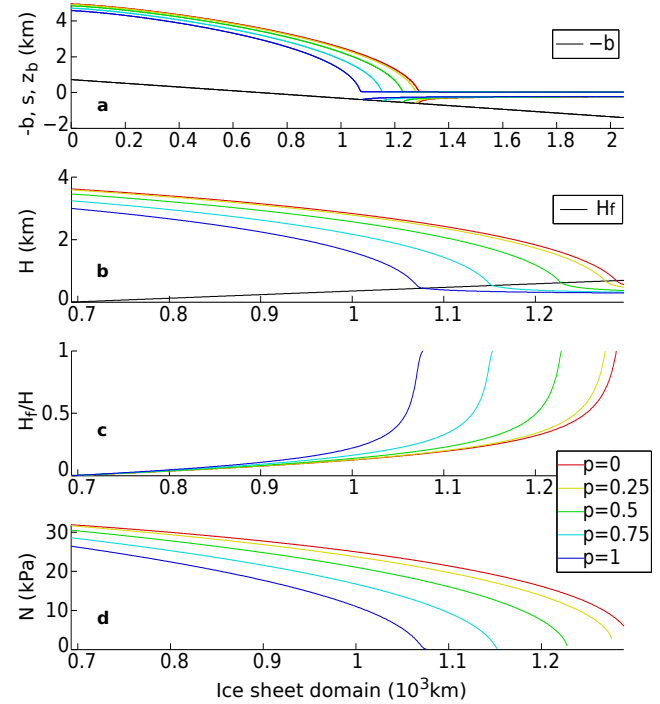

Figure 1. Dependence of ice geometry and effective pressure on the parameter $p$ over a linear bed as in Schoof (2007b). All panels show the fixed-grid solution at $0.8 \mathrm{~km}$ resolution without a grounding-line parameterization (see Sec. 2.3) and with ice softness $A=10^{-25} \mathrm{~Pa}^{-3} \mathrm{~s}^{-1}$. (a) Ice surface and bedrock (colors), and basal elevation (black) over the full ice-sheet domain. (b) Ice sheet thickness (colors) and flotation thickness (black) over the marine portion of the ice sheet. (c) The ratio between the flotation thickness and the ice-sheet thickness. (d) The effective pressure $N$, which approaches zero more smoothly with increasing $p$. Plots in panels (c) and (d) include only grounded cells, as the ice is exactly at flotation and effective pressure is zero elsewhere. The plotted effective pressure does not go to zero for all $p>0$ because the grounding line lies between the last grounded cell and the first floating cell.
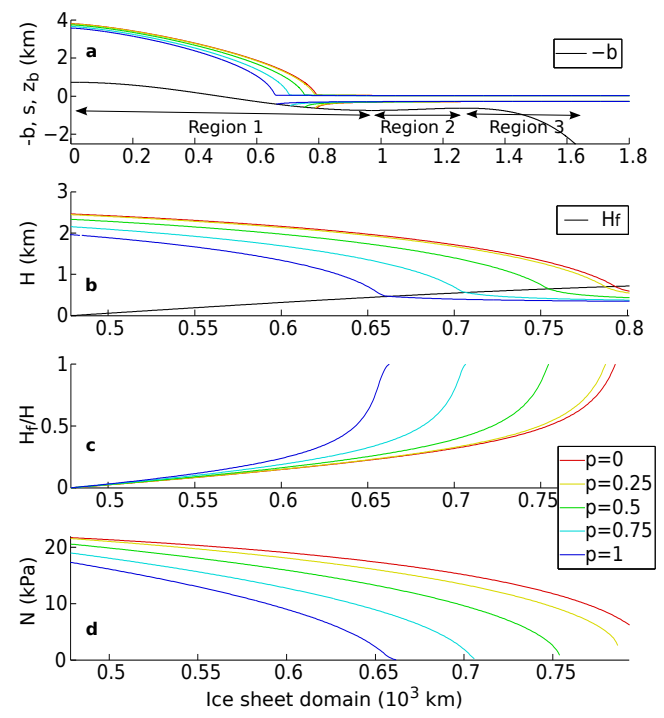

Figure 2. Dependence of ice geometry and effective pressure on $p$, as in Fig. 1 but with the polynomial bed as in Schoof (2007b), containing two stable regions (1 and 3) and an unstable region (2). 
smaller the $p$ value the greater the effective pressure, which tends to move the grounding line seaward. The jump in effective pressure is to be expected for $p=0$ because of the limit defined above. For small values of $p>0$, the transition in basal stress occurs over a narrow region of order $1 \mathrm{~km}$ or less, and is thus resolved only at high model resolution. The figures show that $N$ drops to zero more smoothly as $p$ increases, meaning that the basal stress will also be increasingly smooth.

Parameterized in terms of $p$, Eq. (6) becomes

$$
\tau_{\mathrm{b}}=C|u|^{\frac{1}{n}-1} u\left(\frac{N(p)^{n}}{\kappa|u|+N(p)^{n}}\right)^{\frac{1}{n}},
$$

where $\kappa \equiv \frac{m_{\max }}{\lambda_{\max } A_{\mathrm{b}}}$. This formulation does not require the introduction of an arbitrary length scale of basal transition, as in the parameterization proposed by Pattyn et al. (2006). Equation (15) has two asymptotic behaviors. In the ice sheet interior, the ice is thick and slow-moving, so that $\kappa|u| \ll$ $N(p)^{n}$ and

$\tau_{\mathrm{b}} \approx C|u|^{\frac{1}{n}-1} u$.

In this limit, $\tau_{\mathrm{b}}$ is independent of $p$. Many models define the basal-friction law throughout the ice sheet to have the form of Eq. (16), as in Schoof (2007a) and the MISMIP experiments. This simplified friction law leads to a set of equations with an accurate semi-analytic approximation (Schoof, 2007a, b), whereas the more complex friction law in Eq. (15) does not lend itself to a similar semi-analytic solution (see Appendix B). A boundary-layer solution could be computed numerically, but we have instead opted to compute a highaccuracy benchmark solution over the full domain, as described in the next section. The semi-analytic solution of Schoof (2007a) closely approximates our model as $p$ approaches zero. Figure 3a shows that the basal-stress term (blue) closely matches the limit of high overburden pressure (red) given by Eq. (16) when $p=0$. In this limit, the boundary-layer solution and our high-resolution benchmark solution differ by a few kilometers or less.

The second asymptote, the Coulomb-friction limit, occurs near the grounding line where the ice is thin and fast-flowing, so that $\kappa|u| \gg N(p)^{n}$ and

$\tau_{\mathrm{b}} \approx \frac{C}{\kappa^{\frac{1}{n}}} N(p) \frac{u}{|u|}$.

By construction, when $p=0$ the effective pressure is equal to the full overburden pressure, $p_{\mathrm{i}}$, and the basal stress discontinuously drops to zero across the grounding line. When $p>0$, the effective pressure $N$ smoothly approaches zero at the grounding line over a distance that increases as $p$ increases. Just inland of the grounding line, the basal stress is proportional to the effective pressure.

We define the friction transition zone as the part of the ice sheet where $0 \leq N(p)^{n}<\kappa|u|$, where Coulomb friction is dominant. The friction transition zone is closely related to the transition zone defined in Sect. 1, since the transition from flow dominated by vertical shear to flow dominated by extensional stress must occur in the region where the basal shear stress drops from a large value (high $N$ ) to a small value (low $N$ ). For the range of parameters we studied, the size of the friction transition zone varies between 0 and $\sim 20 \mathrm{~km}$, depending on $p$, the bedrock topography, and the ice softness. Importantly, the size of the friction transition zone is an increasing function of $p$, meaning that, at a given resolution, this zone is better resolved when $p$ is larger. Figure $3 b$ and $3 \mathrm{c}$ show the basal-stress terms and their two asymptotic limits for $p=0.5$ and $p=1$, respectively. Eq. (16), the red curve, dominates in the bulk of the ice sheet, while Eq. (17), shown in green, dominates in the friction transition zone.

The size of the friction transition zone depends on $\kappa \equiv$ $\frac{m_{\max }}{\lambda_{\max } A_{\mathrm{b}}}$ as well as $p$. For this study we chose the values of $m_{\max }, \lambda_{\max }$, and $A_{\mathrm{b}}$ as in Pimentel et al. (2010) and given in Table 2. Since the focus of this paper is on the effect of our effective-pressure parameterization near the grounding line, we defer to a follow-up study a full analysis of how variation of $\kappa$ affects our results at different values of $p$ and $A$. Here we simply summarize what we observed for a specific ice softness $A=4.6416 \times 10^{-25} \mathrm{~Pa}^{-3} \mathrm{~s}^{-1}$. Increasing $\kappa$ by an order of magnitude introduces a finite friction transition zone of $\sim 1 \mathrm{~km}$ when $p=0$ and triples the size of the friction transition zone to $\sim 28 \mathrm{~km}$ when $p=1$. Although the friction transition zone becomes finite when $p=0$, the basal friction remains discontinuous across the grounding line. Even so, a larger value of $\kappa$ could decrease the model resolution required for small values of $p$. Decreasing $\kappa$ by an order of magnitude has no impact on the friction transition zone when $p=0$, but halves the friction transition zone to $\sim 5 \mathrm{~km}$ when $p=1$. More generally, as $\kappa$ goes to zero the basal friction law will asymptote to Eq. (16), regardless of $p$.

Figures 1-3 show that although the friction transition zone is small compared to the whole ice sheet, its effects are farreaching. As $p$ increases from 0 to 1 (other things being equal), the grounding line retreats by more than $100 \mathrm{~km}$, and the steady-state surface elevation is reduced hundreds of $\mathrm{km}$ upstream.

\subsection{Numerics}

Vieli and Payne (2005) and Pattyn et al. (2012) showed that the numerical method used to discretize Eqs. (1)-(4) with a friction law given by Eq. (16) affects model accuracy. They noted that moving-grid models are significantly more accurate at reproducing grounding-line dynamics than fixed-grid models. Although a one-dimensional moving-grid model is easy to implement, moving grids are hard to incorporate in three-dimensional ice-sheet models, whereas fixed grids are well suited for this purpose. To mimic the constraints on realistic 3-D models, we aim to produce a solution of acceptable accuracy using a fixed-grid model with the lowest possible 


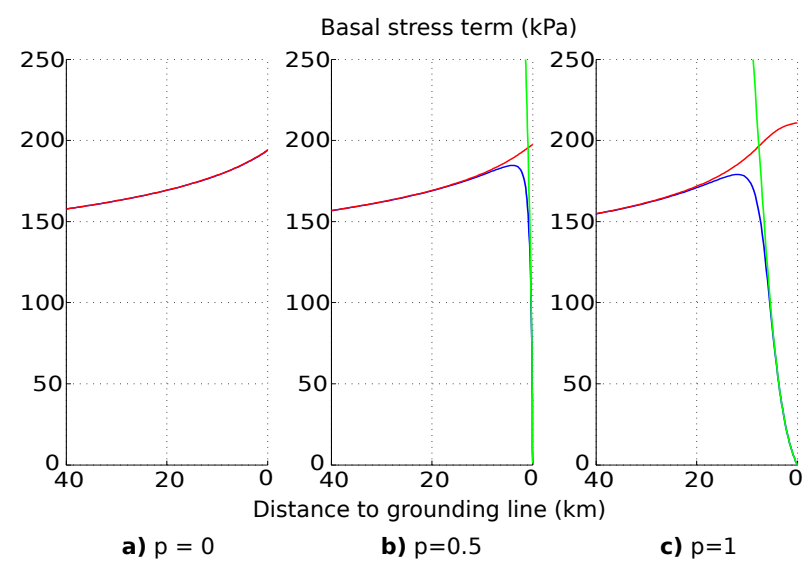

Figure 3. Basal stress given by Eq. (15) (blue) and its asymptotic limits, Eq. (16) (red) and Eq. (17) (green) for ice softness $A=10^{-25} \mathrm{~Pa}^{-3} \mathrm{~s}^{-1}$ and using the Chebyshev benchmark solution. (a) When $p=0$, the second (green) asymptote is never reached, the red and blue curves overlap almost exactly, and there is no friction transition zone (basal stress falls abruptly to zero at the grounding line). (b) and (c) When $p=0.5$ and $p=1$, the length of the friction transition zone, defined as the region where $0 \leq N(p)^{n} \leq \kappa|u|$ (roughly speaking, the region where the blue curve differs from the red curve), ranges from several hundred meters to $20 \mathrm{~km}$ depending on $A, p$ and bedrock topography.

computational cost. As we will show in Sect. 3, depending on the values of the parameter $p$, our parameterization of effective pressure can considerably reduce the computational cost of an accurate fixed-grid simulation.

Pattyn et al. (2006) and Gladstone et al. (2010a) showed that numerical errors (or alternatively, the computational cost of a simulation with a given numerical error) could be reduced through the use of numerical grounding-line parameterizations (GLPs). GLPs involve sub-grid-scale interpolation of the grounding-line position, which is used in the grid cell containing the grounding line to compute a stress that varies continuously as the grounding line moves.

In the following section, we present results both with and without a GLP in order to compare our findings with those of Gladstone et al. (2010a) and to investigate the possible benefit of combining the GLP with our effective-pressure parameterization. We implemented a GLP similar to the PA_GB1 GLP in Gladstone et al. (2010a). First, we determine the grounding-line position based on linear interpolation of the function $f \equiv H_{\mathrm{f}} / H$, given that $f=1$ at the grounding line. Then, in the cell containing the grounding line, we compute the basal and driving stresses once each assuming that the cell is entirely grounded and then entirely floating. Finally, the stresses are linearly interpolated between their grounded and floating values, based on the fraction of the cell that is grounded vs. floating. The resulting expressions for the basal and driving stresses are given by Eqs. (C38) and (C39) respectively. We chose not to use the quadrature methods em-

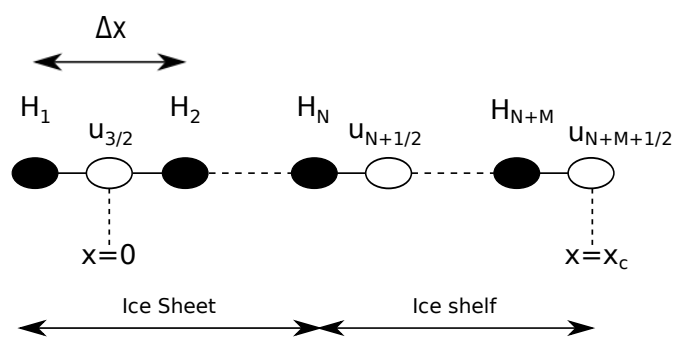

Figure 4. Illustration of the staggered grid used in the model. The $H$-grid points are represented by solid circles and the $u$-grid points by empty circles. $\Delta x$ is the grid spacing (on both $H$-and $u$-grids). $H_{N}$ is the ice thickness in the last grounded point. The ice divide is at $x=0$ and the calving front at $x=x_{\mathrm{c}}$.

ployed in Gladstone et al. (2010a) because they would likely be too cumbersome and costly in 3-D ice-sheet models. In simulations without a GLP, the model computes basal and driving stresses as if the cell containing the grounding line were entirely grounded.

We discretize the equations of motion on a staggered grid, shown in Fig. 4, with alternating velocity and thickness points ( $u$ - and $H$-points). The ice divide $(x=0)$ and the calving front are placed at a $u$-point and an $H$-point, respectively, allowing us to satisfy both boundary conditions naturally. We included a ghost $H$-point to the left of the ice divide to ensure zero surface slope at the divide. The details of the numerics for the fixed-grid model are given in Appendix C1, and a full description of the GLP is given in Appendix C2.

To evaluate the performance of the fixed-grid model, we needed a benchmark solution to compare with our fixed-grid results. To this end we implemented a stretched-grid, pseudospectral method using Chebyshev polynomials (Boyd, 2001) to produce spectrally accurate steady-state benchmark results. The Chebyshev collocation points are non-uniformly distributed over the ice-sheet domain, with the highest resolution at the grounding line and ice divide. Using 1025 Chebyshev modes, the grid spacing continuously decreases from $\sim 80 \mathrm{~m}$ at a distance of $2 \mathrm{~km}$ from the grounding line to $\sim 2.5 \mathrm{~m}$ at the grounding line. We verified the numerical convergence of the Chebyshev benchmark by comparing grounding-line positions with those computed using 2049 modes at various values of $p$ and $A$. We found that results changed by at most $50 \mathrm{~cm}$ when doubling the resolution, suggesting that numerical errors in the Chebyshev groundingline position are negligible compared to those from the fixedgrid model.

We compute errors in our fixed-grid results by comparing them to Chebyshev benchmark solutions. In order to give us further confidence that the benchmark solutions are accurate, we compared the Chebyshev results with the semi-analytic boundary-layer model from Schoof (2007a) known as Model $A$. We can reproduce the grounding-line position in Model A to within fractions of a millimeter if we neglect longitudinal 
stresses, use the friction law from Eq. (16), and apply boundary conditions given by Eqs. (9) and (13). (This approach can be used to reproduce the grounding-line position from Model $A$ but not the velocity and thickness solutions.)

When we included the full longitudinal stress in the Chebyshev model, the differences with the Model A grounding-line position increased to $\sim 1 \mathrm{~km}$. Switching to the more complex basal friction law, Eqs. (14) and (15), introduced further differences of $\sim 1 \mathrm{~km}$ or less. We attribute the differences between Model $A$ and the Chebyshev solution with full longitudinal stress and our friction law to the simplifying assumptions of Model A, rather than to errors in the Chebyshev model. These results give us confidence that the Chebyshev model is producing solutions with errors that should be negligible (of order meters or less) compared to those from the fixed-grid model (order kilometers or more).

This close agreement between the boundary-layer model and our benchmark sheds some light on possible sources of discrepancies between numerical solutions in Pattyn et al. (2012). While some discrepancies are due to numerical error, others are due to different model formulations. The latter is evident in Durand et al. (2009). Whereas they found poor agreement between their Stokes-flow model and the boundary-layer Model B from Schoof (2007a) - which might reflect the differences between a Stokes model and a depthintegrated model - we find excellent agreement between our benchmark (with $p=0$ ) and the boundary-layer model, both of which aim to solve the same equations.

The full details of the method are given in Appendix C3.

\section{Results}

The results described in this section are based on the MISMIP experiments (Pattyn et al., 2012), which are designed to study the transient behavior of marine ice-sheet models. For a given ice softness $A$ we obtain a steady ice-sheet profile. This profile is then used as the initial condition for the next experiment, which evolves to a new steady state with a new value of the ice softness. MISMIP experiment 1 prescribes decreasing values of $A$ and linear bedrock topography, leading to an advancing grounding line. MISMIP experiment 2 is experiment 1 in reverse, where $A$ is increased back to its original value, resulting in grounding-line retreat. Experiment 3 is similar to the combination of experiments 1 and 2, but using a polynomial bedrock topography. A full description of the MISMIP experiments can be found at http://homepages.ulb.ac.be/ fpattyn/mismip/.

Models participating in the MISMIP intercomparison used the friction law of Schoof (2007a), which is equivalent to Eq. (16). For our experiments we test two model configurations, non-GLP and GLP, both of which include the friction law from Eq. (15), with effective pressure defined by Eq. (14). The GLP configuration includes the grounding-line parameterization discussed in Sect. 2.3 and Appendix C2, while the non-GLP configuration does not. We tested five values of the parameter $p$, equally spaced between zero and one, at seven resolutions between 3.2 and $0.05 \mathrm{~km}$, each a factor of two smaller than the previous. Only the results with $p=0$ can be directly compared with the results of Pattyn et al. (2012). By changing $p$ we are changing the physics, not just the numerics, of the problem. Aside from the modified friction law and associated parameterization of effective pressure, we used the standard MISMIP protocols except as specifically stated below.

Typically, differences in grounding-line positions are used to compare the accuracy of ice-sheet model results (Pattyn et al., 2012). This error metric is practical for us as well, since the grounding-line position is easily diagnosed from both Chebyshev and fixed-grid simulations. In realistic simulations, errors in grounding-line position are not as important as those in volume above flotation, which is directly related to the ice sheet's contribution to sea-level change. However, we found (not shown) that the behavior of both metrics is qualitatively similar: larger errors in grounding-line position correspond to larger errors in volume above flotation.

\subsection{Linear-bed experiments}

We performed a series of experiments with the linear bedrock topography of Schoof (2007a), shown in Fig. 1a:

$b(x)=-\left(720-778.5 \frac{x}{750 \mathrm{~km}}\right) \mathrm{m}$.

We forced the ice sheet first to advance and then to retreat by varying the ice softness $A$, in analogy to MISMIP experiments 1 and 2 (Pattyn et al., 2012). To force ice-sheet advance, we incrementally decreased $A$ through the values listed in Table 3, allowing the ice sheet to evolve to steady state each time $A$ was changed. Then, to force retreat, we increased $A$ through the same values in reverse order, again evolving to steady state at each step. Experiments were performed at seven resolutions $(3.2,1.6,0.8,0.4,0.2,0.1$ and $0.05 \mathrm{~km})$, five values of $p(0,0.25,0.5,0.75$ and 1$)$, and both with and without the GLP.

Schoof $(2007 a, b)$ showed that the steady-state groundingline position on a bed sloping monotonically downward in the direction of the ice flow is unique for a given ice softness. Figure 5 shows the grounding-line positions derived from the boundary-layer solution of Schoof (2007a) and those from advance-and-retreat cycles using our Chebyshev and fixed-grid models with $p=0$ at $0.05 \mathrm{~km}$ resolution. The grounding-line position in our Chebyshev simulation differs from that of the boundary-layer solution by less than $1.2 \mathrm{~km}$. As mentioned in the previous section, this difference appears to be mostly due to the fact that the boundary-layer model neglects longitudinal stresses in the bulk of the ice sheet.

The grounding-line position in the fixed-grid model advances relatively accurately, whether or not the GLP is applied, with errors of no more than $1.2 \mathrm{~km}$. During the retreat 


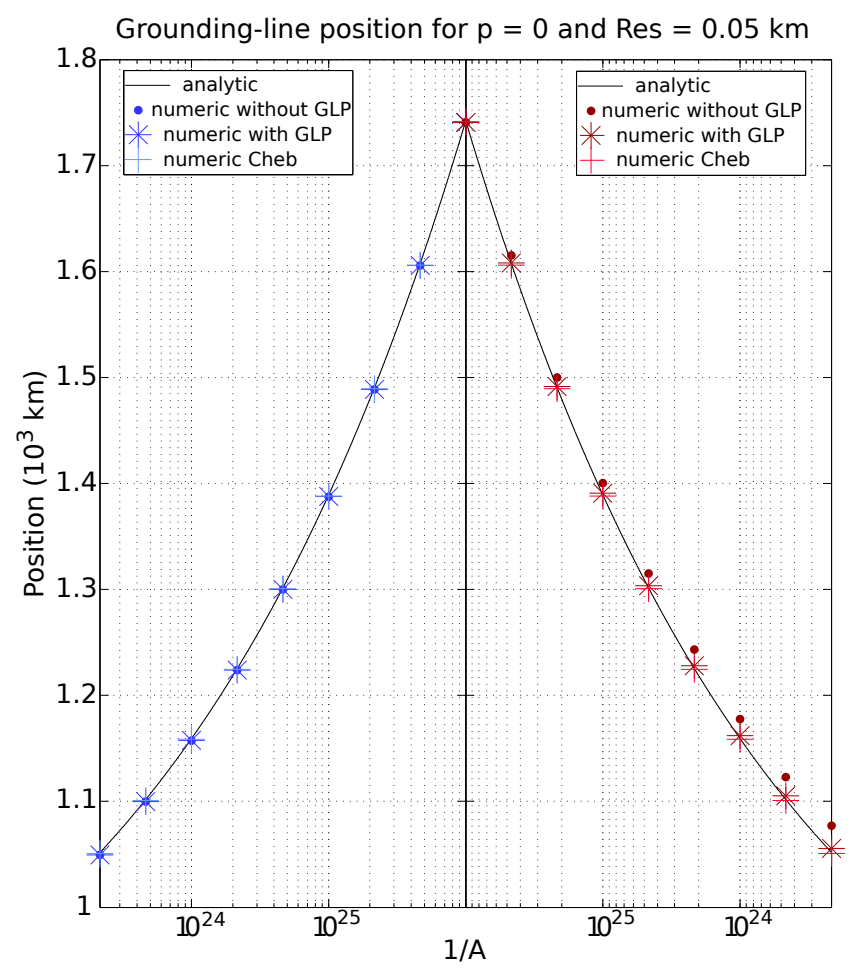

Figure 5. The grounding-line position during advance and retreat experiments over a linear bed at $50 \mathrm{~m}$ resolution with $p=0$ from the boundary-layer solution by Schoof (2007a) (solid black), the Chebyshev benchmark model (pluses), the fixed-grid model without the GLP (dots) and the fixed-grid model with the GLP (stars). The boundary-layer solution is in close agreement with the Chebyshev benchmark (maximum difference of $1.2 \mathrm{~km}$ ), as are the fixedgrid results with or without the GLP. Both fixed-grid models closely agree with the Chebyshev benchmark during advance (maximum difference of $1.2 \mathrm{~km}$ ). During retreat, the model with the GLP matches the benchmark (maximum difference of $5 \mathrm{~km}$ ) better than the model without the GLP (maximum error of $26 \mathrm{~km}$ ).

experiment, the grounding-line position is overestimated as much as $26 \mathrm{~km}$ when the GLP is not used, but by no more than $5 \mathrm{~km}$ with the GLP included, thereby showing its potential benefit.

Figure 6 shows the differences between the grounding-line position from the fixed-grid and benchmark models in several configurations: both without (left) and with (right) the GLP, and at three different resolutions, $1.6 \mathrm{~km}$ (top), $0.4 \mathrm{~km}$ (middle) and $0.1 \mathrm{~km}$ (bottom). We show differences rather than estimated errors (the absolute value of the differences), because the sign of the difference is important in telling whether the grounding line is too far advanced or too far retreated. During the retreat phase of each experiment (the right-hand side of each plot), the fixed-grid grounding line is always too advanced, whereas during the advance phase (the left-hand side of each plot), the grounding line may be too advanced or too retreated depending on the values of $p$ and $A$. For simulations with $p<0.5$ with and without the GLP, the grounding-
Table 3. Values of the ice softness $A$ used in the MISMIP experiment 1 (linear bed). These are the same values prescribed in Pattyn et al. (2012).

\begin{tabular}{cc}
\hline Step no. & $A\left(\times 10^{-26} \mathrm{~s}^{-1} \mathrm{~Pa}^{-3}\right)$ \\
\hline 1 & 464.16 \\
2 & 215.44 \\
3 & 100 \\
4 & 46.416 \\
5 & 21.544 \\
6 & 10 \\
7 & 4.6416 \\
8 & 2.1544 \\
9 & 1 \\
\hline
\end{tabular}

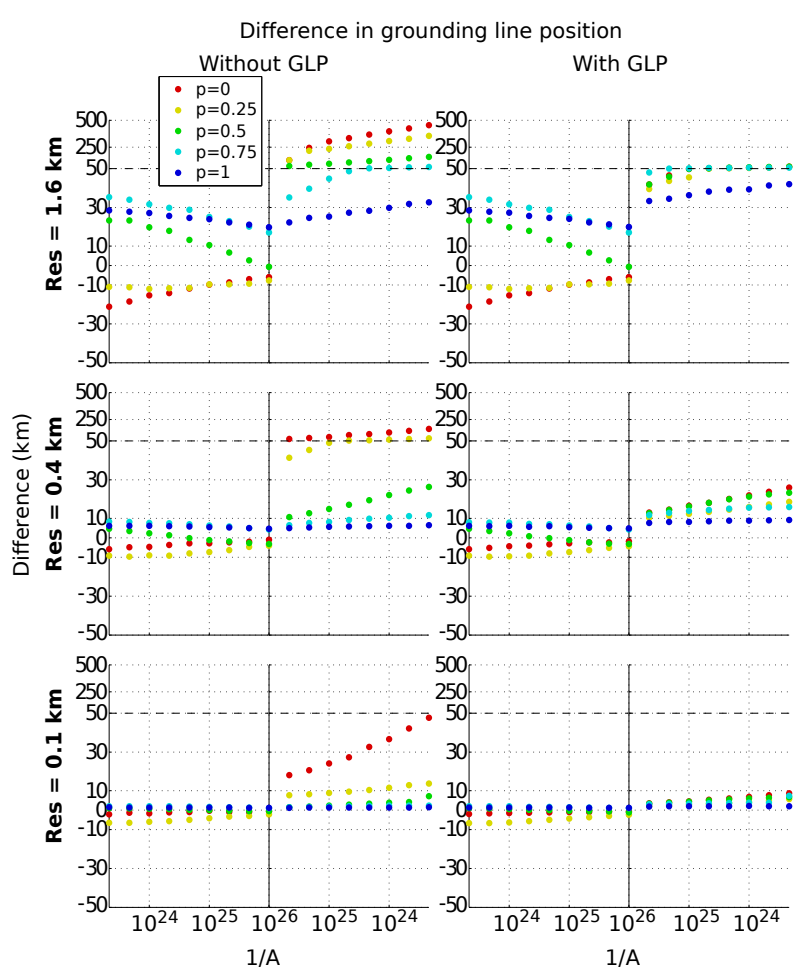

Figure 6. The signed difference between the fixed-grid and benchmark grounding-line positions over a linear bed at $1.6 \mathrm{~km}$ (top row), $0.4 \mathrm{~km}$ (middle row) and $0.1 \mathrm{~km}$ (bottom row) resolution for simulations without GLP (left column) and with GLP (right column). Each column contains both advance (left of each panel) and retreat (right of each panel) experiments. The dashed line (at $50 \mathrm{~km}$ ) in each panel shows the location of a transition in scale of the $y$-axis, which allows the same figure to present both very large and relatively small errors. Errors (the magnitude of the differences) are approximately inversely proportional to the resolution and decrease with increasing $p$, strongly so without the GLP. The GLP reduces the most egregious errors during retreat (occurring when $p$ is small).

line position is not sufficiently advanced during the advance phase. For simulations with $p>0.5$, the grounding-line position is always too advanced during the full experiment. 


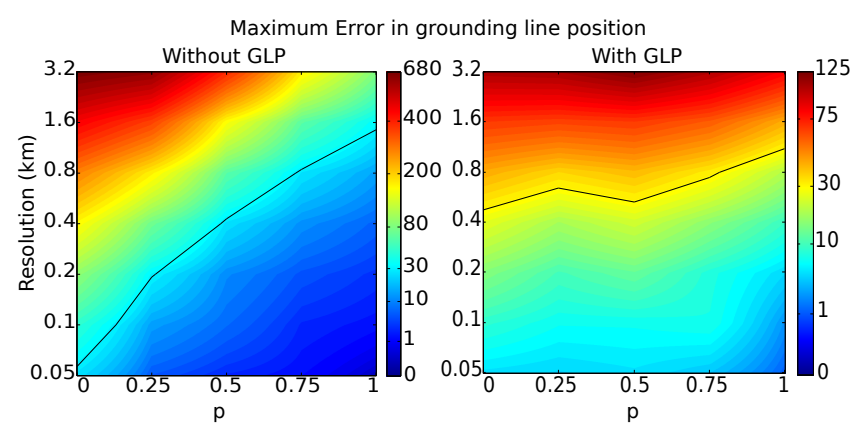

Figure 7. The maximum error over the advance and retreat experiments between the fixed-grid and benchmark grounding-line position over a linear bed for simulation without the GLP (left column) and with the GLP (right column). The errors are bilinear interpolations of our 35 experiments ( 5 values of $p$ and 7 resolutions). The black line shows a contour of $30 \mathrm{~km}$ error $(\sim 5 \%$ of the difference between the most advanced and the most retreated positions of the benchmark), below which we deem the error to be sufficiently small. Note that each panel uses a different nonlinear color bar. Without the GLP, the maximum error decreases approximately linearly with resolution and superlinearly with $p$. With the GLP the maximum error decreases weakly with respect to $p$ but approximately linearly with resolution.

Figure 7 shows the maximum error over an advance-andretreat cycle at a given value of $p$ and resolution without the GLP (left) and with the GLP (right). The error map was obtained by bilinear interpolation from our 35 experiments. The figure shows that the maximum errors decrease approximately linearly with the grid-cell size for each value of $p$, either with or without the GLP. Linear convergence of grounding-line errors with resolution has been seen in other fixed-grid models (Gladstone et al., 2010a; Cornford et al., 2013). Compared with resolution, the application of the GLP and larger values of the parameter $p$ produce a much more dramatic reduction in maximum error.

The differences between experiments are most apparent during the retreat phase of each experiment (right-hand side of each panel in Fig. 6). The experiments most similar to typical MISMIP fixed-grid results - experiments without GLP and with $p=0$ - show huge estimated errors during retreat on the order of hundreds of kilometers. The maximum error is approximately a factor of ten smaller in both the experiments with a GLP at $p=0$ (red dots in the right-hand column) and the experiments without a GLP but with $p=$ 1. Surprisingly, the combination of the GLP and effectivepressure parameterization with $p=1$ does not seem to produce smaller errors than $p=1$ without the GLP, showing diminished performance particularly during retreat. The GLP has essentially no impact on the advance phase (left-hand side of each panel in Fig. 6), whereas the error during advance does tend to decrease as $p$ increases.

The black line in Fig. 7 shows a maximum error in grounding-line position of $\sim 30 \mathrm{~km}(\sim 5 \%$ of the difference between the most advanced and most retreated groundingline positions of the benchmark). We chose this as a (somewhat arbitrary) threshold below which we deem the error to be acceptable. In experiments without the GLP, smoother basal friction (larger values of $p$ ) means that this error threshold has reached a coarser resolution. This is not the case when the GLP is included. Instead we reach our threshold error at roughly the same resolution for all values of $p$. As it turns out, using the GLP is always beneficial when $p \leq 0.5$ but becomes disadvantageous when $p>0.5$.

\subsection{Polynomial-bed experiments}

We performed a second series of grounding-line advanceand-retreat cycles with bedrock topography shown in Fig. 2a and given by the following polynomial function (Schoof, 2007a):

$$
\begin{aligned}
& b(x)=-\left[729-2184.8\left(\frac{x}{750 \mathrm{~km}}\right)^{2}+1031.72\left(\frac{x}{750 \mathrm{~km}}\right)^{4}\right. \\
& \left.-151.72\left(\frac{x}{750 \mathrm{~km}}\right)^{6}\right] \mathrm{m} .
\end{aligned}
$$

These experiments are analogous to MISMIP experiment 3 (Pattyn et al., 2012), but with our modified friction law and effective-pressure parameterization and with more values of the ice softness $A$. The bed topography has three distinct regions. Region 1 slopes downward from the ice divide toward a local minimum, region 2 slopes upward, and region 3 slopes downward again, forming a steep continental-shelf break.

Theoretical arguments (Weertman, 1974; Schoof, 2007a) suggest that stable steady-state grounding-line positions can be found in regions 1 and 3 (with downward-sloping beds) but not in region 2 (with an upward-sloping bed). Our numerical results are consistent with theory. We found that steadystate grounding-line positions do not exist on upward-sloping beds in region 2 but that new steady state solutions are found in region 3 when the grounding line has been forced to advance through region 2 .

Starting with a grounding line in region 1 , we varied the ice softness $A$ to induce grounding-line motion. In his boundary layer model, Schoof (2007a) showed that the grounding-line position exhibits hysteresis: The grounding line jumps across the unstable region at significantly smaller values of $A$ during the advance phase than during the retreat phase. When $p \leq 0.5$ we varied $A$ between $3 \times 10^{-25}$ and $2.5 \times 10^{-26} \mathrm{~s}^{-1} \mathrm{~Pa}^{-3}$, the bounds of MISMIP experiment 3 , over 19 values approximately equally spaced in log space. For values of $p>0.5$, our experiment did not show the full hysteresis behavior within this range of $A$. In fact, for a given ice softness, lower basal friction (i.e., larger $p$ ) tends to move the grounding line inland, as shown in Fig, 2, so that the grounding line never reaches the unstable region for larger values of $p$. In order to obtain hysteresis we extended the 


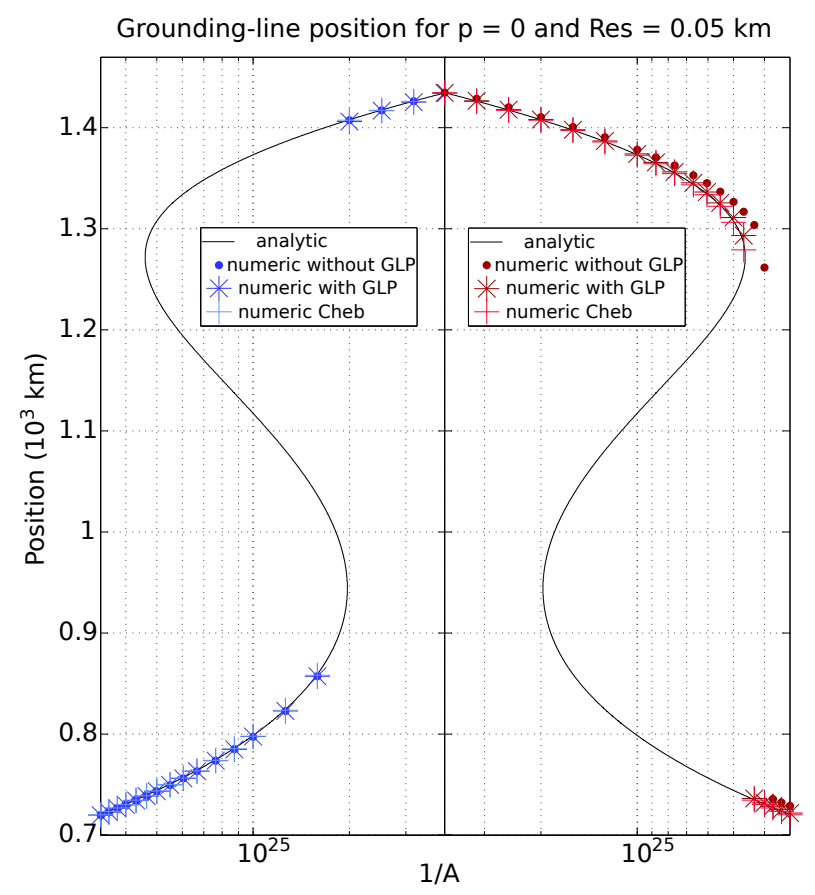

Figure 8. As in Fig. 5 but with the polynomial bed shown in Fig. 2a. The boundary-layer solution closely agrees with Chebyshev results (maximum difference of $1.4 \mathrm{~km}$ ). Fixed-grid results both with and without the GLP closely agree with the Chebyshev benchmark during advance (maximum difference of $\sim 1 \mathrm{~km}$ without the GLP and $\sim 1.6 \mathrm{~km}$ with the GLP). During retreat, the model with GLP is the better match to the benchmark (maximum difference of $\sim 14 \mathrm{~km}$ ). Without the GLP, the fixed-grid grounding-line position matches the benchmark reasonably well when both solutions are on the same side of the unstable region (maximum difference of $\sim 38 \mathrm{~km}$ ) but not in the vicinity of the local maximum where the solutions are on different side of the unstable region, (maximum difference of $\sim 570 \mathrm{~km}$ ).

range of $A$ to $2.5 \times 10^{-27} \mathrm{~s}^{-1} \mathrm{~Pa}^{-3}$ and varied $A$ over 34 values approximately equally spaced in log space. For all experiments, we used more values of $A$ than MISMIP in order to obtain a better statistical sampling of the error within each experiment and to reduce the influence of particularly large errors that occur as the grounding line approaches the unstable region. The largest errors occur when the fixed-grid solution is in region 1 while the benchmark is in region 3 or vice versa.

When $p=0$, the boundary-layer solution, model $A$ of Schoof (2007a), again provides a good approximation of our equations of motion. Figure 8 shows the grounding-line positions derived from the boundary-layer solution together with the positions from experiments using our Chebyshev and fixed-grid models with $p=0$. The grounding line of the boundary-layer solution differs from that in our Chebyshev benchmark simulations by less than $1.4 \mathrm{~km}$, similar to the linear bed experiments. The fixed-grid model at $0.05 \mathrm{~km}$ resolution performs well during the advance phase both with

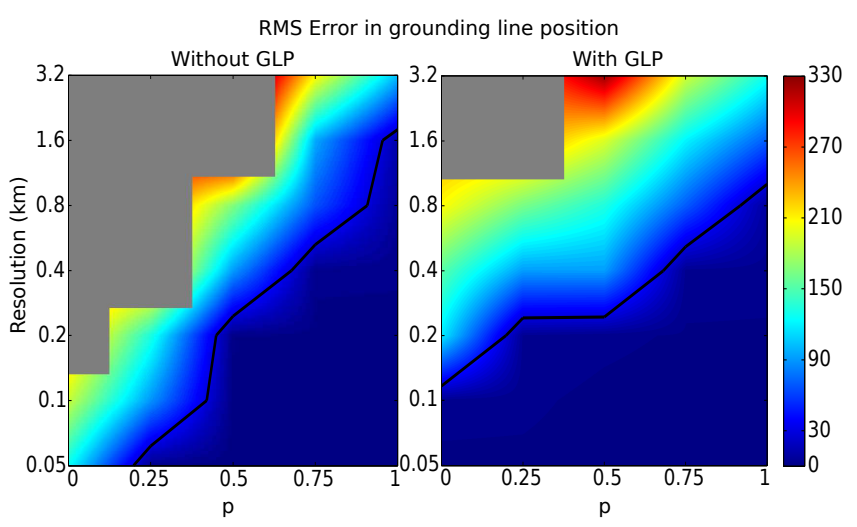

Figure 9. The root-mean-square (RMS) error between the fixedgrid and benchmark grounding-line position over a polynomial bed for simulation without the GLP (left column) and with the GLP (right column). The errors are bilinear interpolations of our 35 experiments. The gray area shows experiments that were not reversible (i.e., for which the fixed-grid grounding line position did not retreat from region 3 to region 1 during the retreat experiment). The black line shows a contour of $30 \mathrm{~km}$ error, below which the error is deemed acceptable, as in Fig. 7. The RMS error without the GLP is approximately inversely proportional to the resolution and decreases strongly with increasing $p$. With the GLP, the RMS error is inversely proportional to the resolution and decreases, though less steeply, with increasing $p$.

and without the GLP; the grounding line is always in the same region (either 1 or 3 ) as the benchmark solution. The maximum error is about $0.9 \mathrm{~km}$ without the GLP and about $1.6 \mathrm{~km}$ with the GLP. However, without the GLP, the model does not perform as well during the retreat phase. The error in the fixed-grid solution is as large as $38 \mathrm{~km}$ when the fixedgrid and benchmark grounding lines are in the same region. The fixed-grid model with the GLP follows the Chebyshev solution more accurately, with a maximum error of $\sim 14 \mathrm{~km}$ and grounding-line positions that always lie in the same region as the benchmark.

During the retreat experiment, the model configuration without GLP shows a grounding-line position located in the wrong region for two values of $\mathrm{A}$, leading to an error of about $570 \mathrm{~km}$. Although this behavior is not seen when the GLP is included, we would likely see similar discrepancies between this configuration and the benchmark if we had sampled an even larger number of $A$ values. In other words, the GLP would appear to reduce the likelihood of these large errors but it is unlikely that they have been eliminated entirely.

The polynomial bed in these experiments represents a more realistic topography than the linear bed. Local maxima and minima, absent in the linear topography, have a significant impact on both model dynamics and numerical errors. Figure 9 shows the root-mean-square (RMS) error between the fixed-grid and benchmark grounding-line position for simulation without the GLP (left plot) and with the GLP (right plot). The error map was obtained by bilinear 
interpolation from our 35 experiments. Here, we used the RMS error instead of the maximum error because the latter is typically dominated by cases in which the benchmark and fixed-grid grounding lines lie in different regions and is highly sensitive to the particular choice of $A$ values. The gray area in each panel indicates experiments which are not reversible (the fixed-grid grounding line position fails to retreat back to region 1 at the end of the retreat experiment). The figure shows that, without the GLP, the RMS error decreases linearly with resolution and rapidly with increasing $p$. When the GLP is included, the RMS error decreases linearly with resolution, while increasing $p$ has a less dramatic impact. As was the case for the linear bed experiments, including the GLP improves the error for small values but not necessarily for large values of $p$. For small $p$, including the GLP improves the ability of the model to retreat past the unstable region, as shown by the reduced grey area on the right-hand side of Fig. 9. All experiments with the GLP and a resolution of $\sim 1 \mathrm{~km}$ or higher show reversibility, whereas a resolution of between 100 and $200 \mathrm{~m}$ is required without GLP when $p=0$.

Similarly to the previous section, we use a threshold of $30 \mathrm{~km}$ as the maximum allowable RMS error, indicated by the black contour line in Fig. 9. Our results show that even with the GLP, resolution as high as $100 \mathrm{~m}$ is required in locations with large effective pressure near the grounding line $(p \sim 0)$. On the other hand, a resolution of $\sim 1 \mathrm{~km}$ is sufficient where the effective pressure is low $(p \sim 1)$. In general, the results from the linear section remain valid when using a polynomial bed: When $p \leq 0.5$, using the GLP leads to smaller RMS errors and better reversibility. However, when $p>0.5$, using a GLP is disadvantageous.

Figure 10 shows the error in grounding-line position as a function of the benchmark grounding-line position during the retreat phase. The figure makes clear that the error increases as the grounding line approaches the unstable region. These results suggest that the fixed-grid model can capture hysteresis with increasing fidelity as $p$ increases and (to a lesser extent) as resolution increases, and that errors nearly always decrease at a given value of $x_{\mathrm{g}}$ as $p$ increases.

Figure 10 also suggests that errors may be a strong function of bedrock slope. The largest errors occur near the local maximum in bed elevation at around $x=1.25 \times 10^{3} \mathrm{~km}$, and decrease sharply as the bedrock steepens further into region 3. This behavior is to be expected as the grounding line approaches a bifurcation point. No stable steady-state solution will exist near that maximum if $A$ is decreased further; small changes in $A$ will lead to large changes in grounding-line position. Similar inverse correlation between bed slope and error can be seen in region 1, though the bedrock steepens more gradually in this region.

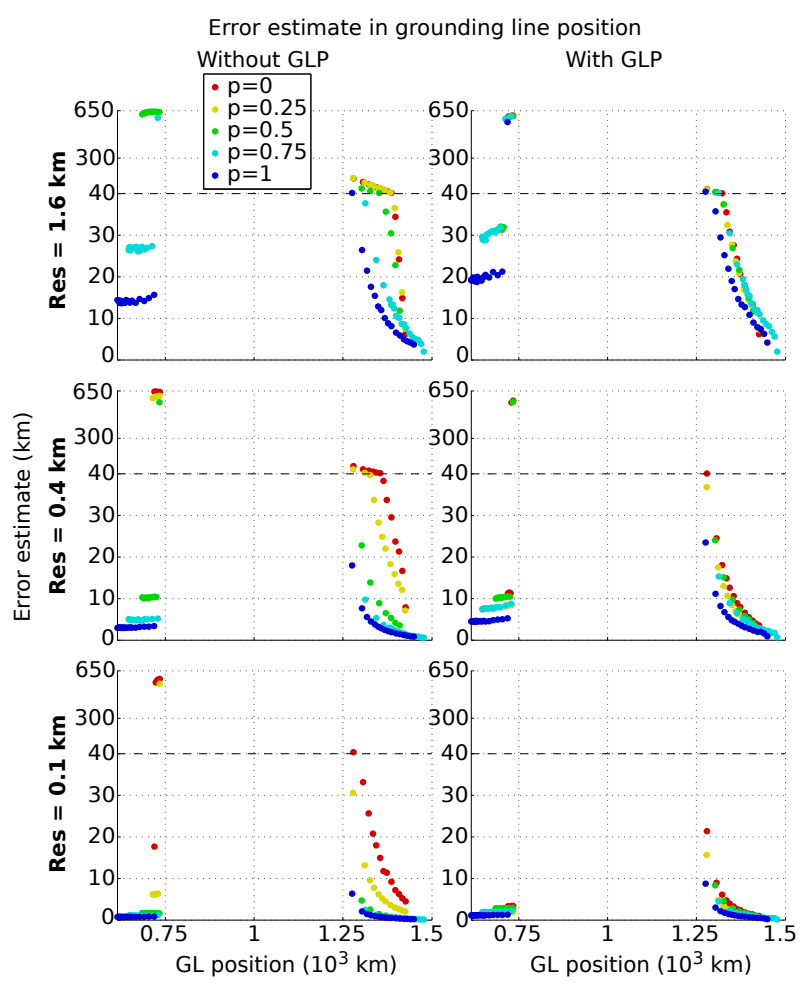

Figure 10. As in Fig. 6, but showing only the retreat experiment over the polynomial bed and with benchmark grounding-line position instead of $A$ along the $x$-axis. The dashed line (at $40 \mathrm{~km}$ ) in each panel shows the location of a transition in scale of the $y$ axis used to show large errors without losing the differences between smaller errors. The error in grounding-line position is consistently lower at a given grounding-line position when $p=1$ than for smaller values of $p$. The figure shows that errors tend to increase as the grounding line approaches the unstable region (empty gap in each figure).

\section{Discussion}

Previous studies of marine ice-sheet dynamics have suggested that grounding-line position converges with increasing resolution (Vieli and Payne, 2005), typically linearly (Gladstone et al., 2010a; Cornford et al., 2013). We find this to be true in our simulations. However, we do not observe the numerical instability seen at coarser resolution in Gladstone et al. (2010a) or the premature retreat found in the fixed-grid model of Goldberg et al. (2009), as seen in their Fig. 4b. Instead, with the use of our basal-friction parameterization and assuming good connectivity to the ocean $(p \sim 1)$, we find that a fixed-grid model can yield accurate results at relatively coarse resolution. These improvements do not require modified numerical techniques, such as a GLP, but arise from plausible changes in model physics.

Assuming these results extend to three-dimensional models, the implications are significant. In our experiments the grounding-line position converges at $\sim 1 \mathrm{~km}$ grid resolution 
or coarser when the basal shear stress smoothly approaches zero near the grounding line. Much finer resolution, $\sim 500 \mathrm{~m}$ in the linear bed experiment and $\sim 100 \mathrm{~m}$ in the polynomial bed experiment, is required when the basal stress is discontinuous at the grounding line. More realistic models using discontinuous basal stress have shown accurate groundingline migration only with a resolution of $200 \mathrm{~m}$ or less (Cornford et al., 2013), consistent with our simulations. Our results suggest that it may be possible to simulate marine ice sheets at much lower computational expense than would be required with traditional friction laws. Models with adaptive and unstructured grids (Goldberg et al., 2009; Favier et al., 2012; Perego et al., 2012; Cornford et al., 2013) could be made more computationally efficient by reducing the need for very fine resolution near grounding lines. Also, our parameterization might allow uniform-grid models to simulate whole ice sheets, since $\sim 1 \mathrm{~km}$ resolution throughout the ice sheet is feasible (though expensive). However, this could require setting $p \sim 1$ everywhere in the ice sheet, which might not be physically realistic for some regions.

Our one-dimensional model has several simplifying assumptions that may limit its applicability to real ice sheets. Notably, the model does not include vertical shear stress (so it cannot simulate flow over a frozen bed) or lateral shear stress (so it does not include effects of ice-shelf buttressing). These missing stresses are likely to be large enough (Whillans and van der Veen, 1997; Schoof, 2007a) that we cannot validate our results by direct comparison to observations. In particular, Goldberg et al. (2009) showed, in a series of experiments with basal stress corresponding to ours when $p=0$, that buttressing can affect the rate and direction of grounding-line advance or retreat over upward-sloping beds. Gladstone et al. (2012) showed that buttressing can relax the requirement for high resolution, so that without buttressing, a model always requires higher resolution than if buttressing is included. This may imply that our results can be considered an upper bound in model resolution requirement.

Most large-scale ice-sheet models do not explicitly model basal hydrology, but instead use inversion to compute a spatially variable basal sliding coefficient based on observations. (The basal sliding coefficient is typically equivalent to $C$ in Eq. 16). In some cases the basal sliding coefficient obtained by inversion decreases to zero at or near the grounding line (Vieli and Payne, 2003; Larour et al., 2012), suggesting that (in the terms of our model) $p>0$. In ice-sheet models that invert for a spatially varying parameter $C$ (or its equivalent), the inversion process will tend to find a value of $C$ that is close to zero near the grounding line in regions with significant basal-water support, leading to an initial $\tau_{\mathrm{b}}$ that is continuous (or nearly continuous) across the grounding-line. However, in the absence of a basal-friction law that responds to changes in the grounding-line location, the grounding line will migrate over time but $C$ will remain fixed in space. This is likely to lead to large jumps in basal stress across the grounding line at later times, and to non- convergent grounding-line dynamics at coarser resolution. An advantage of our parameterization is that, for larger values of $p$, the basal stress remains continuous and smooth over a resolvable friction transition zone even as the grounding line moves.

We plan to incorporate our parameterization in the Community Ice Sheet Model (CISM), a three-dimensional model with support for a variety of higher-order stress approximations, several types of grids, and coupling to global climate models (Rutt et al., 2009; Perego et al., 2012; Lipscomb et al., 2013). A key challenge is to choose realistic values of $p$. One approach would be to invert for $p$ using present-day data and obtain a map of $p$. From this map we could derive average values of $p$ for specific regions or for the entire Antarctic ice sheet. It is not clear, however, that setting $p$ to a large value everywhere would give an acceptable simulation. Large $p$ would reduce the requirement for high grid resolution, but could also yield ice sheets that are smaller than observed in regions where the basal friction does, in fact, make a sharp transition near the grounding line (i.e., where $p$ is small). It might be possible to compensate for such errors by adjusting other parameters, but only at the cost of physical realism.

This study has focused on the sensitivity of grounding-line dynamics to variations in the effective-pressure parameter $p$. Other model parameters also affect the dynamics (Gladstone et al., 2012): for example, the inland asymptotic value $N=\rho_{i} g H$ in Eq. (14), the constants $C$ and $\kappa$ in Eq. (15), the bed slope, and (if lateral drag were parameterized in the model) the channel width. In future work we will investigate the model sensitivity to changes in these parameters.

Another limitation of this study is the focus on steadystate solutions. The Chebyshev code used for this paper to obtain benchmark solutions is capable only of computing steady states. We have recently developed a time-dependent (but much slower) code that could be used to benchmark the model's transient behavior. We could then study the effects of our parameterization (with or without the GLP) on short time scales (e.g., decades) that are of great practical interest.

\section{Conclusions}

Applying the MISMIP benchmark experiments to a onedimensional, vertically-integrated, fixed-grid model, we have shown several advantages of a new effective-pressure parameterization together with an appropriate basal-friction law. The parameterization regularizes the ice-flow equations by allowing the basal shear stress beneath grounded ice to decrease smoothly to zero near the grounding line. Physically, it can be viewed as a simple representation of hydrological connectivity between the subglacial water system and the ocean. The parameter $p$ controls the degree of connectivity, ranging from zero (high effective pressure near the grounding line, with no water pressure support from the ocean) to one (low effective pressure near the grounding line, with full 
water pressure support from the ocean). For larger values of $p$, the friction transition zone extends farther from the grounding line, reducing the need for very high grid resolution. Steady-state model results converge to a given error tolerance at much coarser resolutions with a smoothly varying basal shear stress than with a discontinuous stress $(p=0)$.

We found that a numerical grounding-line parameterization (GLP) can greatly reduce errors in grounding-line dynamics in cases where effective pressure is large and basal friction is discontinuous $(p \sim 0)$ but that the GLP slightly increases errors when the basal friction is smooth $(p \sim 1)$. For the MISMIP experiments we chose an error threshold of $30 \mathrm{~km}$ in the grounding-line position. Without a GLP the required grid resolutions are $\sim 1.5 \mathrm{~km}$ when $p=1$, but $<100 \mathrm{~m}$ when $p=0$. With a GLP the required resolutions when $p=1$ are again $\sim 1 \mathrm{~km}$, compared to $500 \mathrm{~m}$ (for the linear bed) and $100 \mathrm{~m}$ (for the polynomial bed) when $p=0$. Given that it would be impractical to use a GLP in some regions but not others based on the smoothness of the local basal friction, our results suggest that, on balance, inclusion of the GLP is likely to reduce grounding-line errors.
Our effective-pressure parameterization is by no means a sophisticated hydrology or till model. It represents only the part of the hydrological system that is connected to the ocean and reaches ocean pressure at the grounding line. In our experiments the parameter $p$ affects basal sliding within $\sim 20 \mathrm{~km}$ of the grounding line, where ocean water pressure is a significant fraction of the ice overburden pressure. A more detailed model would predict the evolution of subglacial conduits and till, probably expanding the region where subglacial water supports much of the overburden. Compared to our parameterization, such a model would more realistically simulate how effective pressure varies upstream of the grounding line, giving a more accurate treatment of grounding-line migration. If the model predicted hydrological connectivity near the grounding line, its simulated basal friction could qualitatively resemble the friction given by our simple model, reducing the requirement for very high resolution. 


\section{Appendix A: Basal water pressure inland of the grounding line}

Here we show that the basal water pressure approaches a fraction $p$ of the ocean water pressure inland of the grounding line (in the limit $H_{f} \ll H$ ). We combine the definition of the effective pressure, $N \equiv p_{\mathrm{i}}-p_{\mathrm{w}}$, and the overburden pressure, $p_{\mathrm{i}} \equiv \rho_{\mathrm{i}} g H$, with Eq. (14) to solve for the basal water pressure $p_{\mathrm{w}}$ :

$p_{\mathrm{w}}=\rho_{\mathrm{i}} g H\left[1-\left(1-\frac{H_{\mathrm{f}}}{H}\right)^{p}\right]$.

In the limit $H_{f} \ll H$, this expression can be approximated by the first term in the Taylor series

$$
\begin{aligned}
p_{\mathrm{w}} & \sim \rho_{\mathrm{i}} g H\left(p \frac{H_{\mathrm{f}}}{H}\right), \\
& \sim p \rho_{\mathrm{i}} g H_{\mathrm{f}},
\end{aligned}
$$

which is $p$ times the ocean water pressure at the depth of the bed, $p_{\text {ocean }}=\rho_{\mathrm{w}} g b=\rho_{\mathrm{i}} g H_{\mathrm{f}}$.

\section{Appendix B: Our effective-pressure parameterization in a boundary-layer formulation}

This section was written based on suggestions from the anonymous reviewer of the paper.

We can rewrite the boundary layer formulation from Schoof (2007b) using our more complex basal friction law:

$(U H)_{x}=0$,

$$
\begin{aligned}
& 4\left(H\left|U_{x}\right|^{\frac{1}{n}-1} U_{x}\right)_{x}-\left(1+\frac{v|U|}{H^{n}\left(1-\frac{H_{f}}{H}\right)^{n p}}\right)^{-\frac{1}{n}} \\
& \qquad|U|^{\frac{1}{n}-1} U-H H_{x}=0, \\
& 4 H\left|U_{x}\right|^{\frac{1}{n}-1} U_{x}=\frac{\delta}{2} H_{f}^{2} \text { at } X=0, \\
& H=H f \text { at } X=0, \\
& U H \rightarrow 0 \text { as } X \rightarrow-\infty, \\
& U \rightarrow 0 \text { as } X \rightarrow-\infty,
\end{aligned}
$$

where $v=\frac{\kappa[U]}{\rho_{i} g[H]}$ and $[U]$ and $[H]$ are the characteristic velocity and thickness scales, respectively, in the boundary layer. Equations (B1)-(B6) are identical to the original boundary-layer model in Schoof (2007b) except for the additional term appearing in the basal friction law:

$\gamma \equiv\left(1+\frac{\nu|U|}{H^{n}\left(1-\frac{H_{f}}{H}\right)^{n p}}\right)^{-\frac{1}{n}}$.

The outer solution remains unchanged, as $\gamma \rightarrow 1$ when $H \rightarrow$ $\infty$.

Unless $v$ is large, the boundary layer size remains the same as the one in Schoof (2007b). Otherwise the boundary layer width would significantly exceed the boundary layer scale estimated in Schoof (2007b). Because of the complexity of the factor $\gamma$, the flux $Q=U H$ can no longer be expressed as a power law in $H_{f}$, as in Schoof (2007a) and Schoof (2007b), meaning that the boundary-layer formulation cannot be computed analytically. We could solve these equations, together with the outer problem, numerically but we have chosen instead to solve the unsimplified equations throughout the domain using a Chebyshev code that focuses resolution near the grounding line (in the boundary layer). 


\section{Appendix C: Numerical methods}

Here we describe the numerical methods behind the fixedgrid, finite-difference model and the stretched-grid Chebyshev model. We hope this will facilitate comparison with other modeling algorithms. In what follows, we denote vectors with bold italics and matrices with bold capital letters.

\section{C1 Fixed-grid model without a GLP}

In the fixed-grid model, we used staggered finite difference to solve Eqs. (1)-(4), (7)-(9), (13) and (15), rewritten here for convenience:

$$
\begin{aligned}
& H_{\mathrm{t}}+(u H)_{x}=a, \\
& 2 A^{-\frac{1}{n}}\left(H\left|u_{x}\right|^{\frac{1}{n}-1} u_{x}\right)_{x}-C|u|^{\frac{1}{n}-1} u\left(\frac{N(p)^{n}}{\kappa|u|+N(p)^{n}}\right)^{\frac{1}{n}} \\
& -\rho_{\mathrm{i}} g H(H-b)_{x}=0 \text {, } \\
& u=0 \\
& (H-b)_{x}=0 \\
& H=\frac{\rho_{\mathrm{w}}}{\rho_{\mathrm{i}}} b \\
& \text { at } x=0 \text {, } \\
& \text { at } x=0 \text {, } \\
& \text { at } \quad x=x_{\mathrm{g}} \text {. } \\
& 2 A^{-\frac{1}{n}}\left|u_{x}\right|^{\frac{1}{n}-1} u_{x}-\frac{1}{2} \rho_{\mathrm{i}}\left(1-\frac{\rho_{\mathrm{i}}}{\rho_{\mathrm{w}}}\right) g H=0 \quad \text { at } \quad x \geq x_{\mathrm{g}} .
\end{aligned}
$$

We use centered differences to discretize Eqs. (C2)-(C6) on a uniform grid. To insure numerical stability, we use a firstorder upwinding scheme and a semi-implicit time stepping scheme to discretize Eq. (C1).

We compute $u$ and $H$ on staggered grids separated by half a grid cell, as shown on Fig. 4. The ice-sheet-ice-shelf domain contains $N+M$ points, where $N$ is the number of points in the ice sheet (changing in time as the grounding line migrates) and $M$ the number of points in the ice shelf on both the $H$-grid.

Since the boundary conditions in Eq. (C3) and (C4) are most easily satisfied at a $u$-grid point, we place the ice divide, $x=x_{\mathrm{d}}=0$, at the first point on the $u$-grid. In general, the grounding-line position, $x=x_{\mathrm{g}}$, lies between two grid points and is diagnosed from $H$ using Eq. (C5), the flotation condition. The boundary condition given by Eq. (C6) applies in the entire ice shelf domain.

We found that it simplified computations near the ice divide to place a "ghost" $H$-grid point to the left of the divide; we enforce symmetry by requiring that the ice thickness is symmetric across the ice divide, that is $H_{1}=H_{2}$, satisfying Eq. (C4). Similarly, we find that a ghost point beyond the calving-front, this time on the $u$-grid, makes it easier to simultaneously solve Eq. (C2) at the last "real" $u$-grid point and Eq. (C6) at the calving front. This ghost point is also needed to solve Eq. (C1) at the calving front.

Excluding the two half cells associated with these ghost points, there are $2(N+M)-3$ half cells between the ice divide and the calving front on the staggered grid. Thus, the spacing between points on both the $H$ - and $u$-grids is given by

$$
\Delta x=\frac{L}{N+M-\frac{3}{2}},
$$

where $L=x_{\mathrm{c}}-x_{\mathrm{d}}$ denotes the length of the domain.

For an integer index $i \in[1, N+M]$, we define the location of $H$-grid points by $x_{i} \equiv(i-3 / 2) \Delta x$ and those of $u$ grid points by $x_{i+1 / 2} \equiv(i-1) \Delta x$. Similarly, we introduce a time index $j \in[0, T]$, where $T$ is the number of time steps in a given simulation, so that $t_{j}=j \Delta t$ for a constant time step $\Delta t$.

Discrete values of thickness and velocity, are $H_{i}^{j} \equiv$ $H\left(x_{i}, t_{j}\right)$ and $u_{i+1 / 2}^{j} \equiv u\left(x_{i+1 / 2}, t_{j}\right)$, respectively. The grounding line position is defined as $x_{\mathrm{g}}^{j}=x_{\mathrm{g}}(j \Delta t)$. The depth of the ice-sheet bed is defined as $b_{\mathrm{i}} \equiv b\left(x_{i}\right)$ at $H$-points and by $b_{\mathrm{g}}=b\left(x_{\mathrm{g}}\right)$ at the grounding line. The effective pressure, $N(x, t ; p)$, is located on an $H$-grid point and is defined by $\mathcal{N}_{i}^{j} \equiv N\left(x_{i}, t_{j} ; p\right)$ (not to be confused with the number of ice-sheet grid points $N)$ :

$\mathcal{N}_{i}^{j}=\rho_{\mathrm{i}} g H_{i}^{j}\left(1-\frac{H_{f_{i}}}{H_{i}^{j}}\right)^{p}$.

The flotation thickness, $H_{\mathrm{f}}$, is defined at $H$-grid points as

$H_{f_{i}} \equiv H_{\mathrm{f}}\left(x_{i}\right)=\max \left(0, b_{\mathrm{i}} \rho_{\mathrm{w}} / \rho_{\mathrm{i}}\right)$.

Equation (C1) is discretized at $H$-grid points throughout the domain (both ice sheet and ice shelf):

$$
\frac{H_{i}^{j+1}-H_{i}^{j}}{\Delta t}+\theta \mathcal{F}_{i}^{j+1}+(1-\theta) \mathcal{F}_{i}^{j}=a,
$$

where

$\mathcal{F}_{i}^{j} \equiv \frac{\left(H_{\text {up }, i+\frac{1}{2}}^{j} u_{i+\frac{1}{2}}^{j}-H_{\text {up }, i-\frac{1}{2}}^{j} u_{i-\frac{1}{2}}^{j}\right)}{\Delta x}$,

and where we have used first-order upwinding, with the upwind thickness defined by

$$
H_{\text {up }, i+\frac{1}{2}}^{j}= \begin{cases}H_{i}^{j} & \text { if } u_{i+\frac{1}{2}}^{j} \geq 0, \\ H_{i+1}^{j} & \text { if } u_{i+\frac{1}{2}}^{j} \leq 0 .\end{cases}
$$

The time centering is determined by $0 \leq \theta \leq 1$ : If $\theta=1$, the time stepping is fully implicit; if $\theta=0$, we are using a fully explicit scheme; and if $\theta=1 / 2$, the method is the partially implicit, second-order accurate in time CrankNicholson scheme.

Equation (C2) is most naturally discretized at $u$-grid points, requiring that the thickness, $H$, in the driving stress and the effective pressure, $\mathcal{N}$, in the friction law be averaged 
to the $u$-grid, e.g., $H_{i+\frac{1}{2}}^{j}=\left(H_{i}^{j}+H_{i+1}^{j}\right) / 2$. In the grounded ice sheet, Eq. (C2) in discrete form is

$$
\begin{aligned}
& \frac{2 A^{-\frac{1}{n}}}{\Delta x^{1+\frac{1}{n}}}\left[H_{i+1}^{j}\left|u_{i+\frac{3}{2}}^{j}-u_{i+\frac{1}{2}}^{j}\right|^{\frac{1}{n}-1}\left(u_{i+\frac{3}{2}}^{j}-u_{i+\frac{1}{2}}^{j}\right)\right. \\
& \left.-H_{i}^{j}\left|u_{i+\frac{1}{2}}^{j}-u_{i-\frac{1}{2}}^{j}\right|^{\frac{1}{n}-1}\left(u_{i+\frac{1}{2}}^{j}-u_{i-\frac{1}{2}}^{j}\right)\right] \\
& -C\left|u_{i+\frac{1}{2}}^{j}\right|^{\frac{1}{n}-1} u_{i+\frac{1}{2}}^{j}\left[\frac{\left(\frac{\mathcal{N}_{i+1}^{j}+\mathcal{N}_{i}^{j}}{2}\right)^{n}}{\kappa\left|u_{i+\frac{1}{2}}^{j}\right|+\left(\frac{\mathcal{N}_{i+1}^{j}+\mathcal{N}_{i}^{j}}{2}\right)^{n}}\right]^{\frac{1}{n}} \\
& -\rho_{\mathrm{i}} g \frac{\left(H_{i+1}^{j}+H_{i}^{j}\right)}{2} \frac{\left(H_{i+1}^{j}-b_{i+1}-H_{i}^{j}+b_{\mathrm{i}}\right)}{\Delta x}=0 .
\end{aligned}
$$

In the ice shelf, Eq. (C2) is discretized as

$$
\begin{aligned}
& \frac{2 A^{-\frac{1}{n}}}{\Delta x^{1+\frac{1}{n}}}\left[H_{i+1}^{j}\left|u_{i+\frac{3}{2}}^{j}-u_{i+\frac{1}{2}}^{j}\right|^{\frac{1}{n}-1}\left(u_{i+\frac{3}{2}}^{j}-u_{i+\frac{1}{2}}^{j}\right)\right. \\
& \left.-H_{i}^{j}\left|u_{i+\frac{1}{2}}^{j}-u_{i-\frac{1}{2}}^{j}\right|^{\frac{1}{n}-1}\left(u_{i+\frac{1}{2}}^{j}-u_{i-\frac{1}{2}}^{j}\right)\right] \\
& -\rho_{\mathrm{i}} g \frac{\left(H_{i+1}^{j}+H_{i}^{j}\right)}{2} \frac{\left(H_{i+1}^{j}-H_{i}^{j}\right)}{\Delta x}=0 .
\end{aligned}
$$

The boundary conditions, Eqs. (C3), (C4) and (C6), are expressed as

$$
\begin{array}{ll}
u_{\frac{3}{2}}^{j} & =0, \\
\frac{\left(H_{2}^{j}-b_{2}-H_{1}^{j}+b_{1}\right)}{\Delta x} & =0,
\end{array}
$$

$\frac{1}{\Delta x^{\frac{1}{n}}} 2 A^{-\frac{1}{n}}\left|u_{N+M+\frac{1}{2}}^{j}-u_{N+M-\frac{1}{2}}^{j}\right|^{\frac{1}{n}-1}$

$$
\left(u_{N+M+\frac{1}{2}}^{j}-u_{N+M-\frac{1}{2}}^{j}\right)-\frac{1}{2} \rho_{\mathrm{i}}\left(1-\frac{\rho_{\mathrm{i}}}{\rho_{\mathrm{w}}}\right) g H_{N+M}^{j}=0 .
$$

We solve the stress-balance equation for $u_{i+1 / 2}^{j+1}$ and the continuity equation for $H_{i}^{j+1}$ using Picard fixed-point iteration. We use the thickness and velocity from the previous time step as the initial guesses:

$H_{i}^{j, 0} \equiv H_{i}^{j-1}$

$u_{i+1 / 2}^{j, 0} \equiv u_{i+1 / 2}^{j-1}$,

At each iteration, we first solve for the new velocity $u_{i+1 / 2}^{j, k+1}$ from the stress-balance equation using the "iterate on viscosity" method which can be found in Goldberg et al. (2009).
The longitudinal-stress term $\left(T_{l} \equiv\left(H \tau_{l}\right)_{x}\right)$ at iteration $k+1$ is defined as

$$
\begin{aligned}
T_{l, i+\frac{1}{2}}^{j, k+1} \equiv & \frac{1}{\Delta x^{2}}\left[H_{i+1}^{j, k} v_{i+1}^{j, k}\left(u_{i+\frac{3}{2}}^{j, k+1}-u_{i+\frac{1}{2}}^{j, k+1}\right)\right. \\
& \left.-H_{i}^{j, k} v_{i}^{j, k}\left(u_{i+\frac{1}{2}}^{j, k+1}-u_{i-\frac{1}{2}}^{j, k+1}\right)\right], \\
v_{i}^{j, k} \equiv & \frac{2 A^{-\frac{1}{n}}}{\Delta x^{\frac{1}{n}-1}}\left|u_{i+\frac{1}{2}}^{j, k}-u_{i-\frac{1}{2}}^{j, k}\right|^{\frac{1}{n}-1} .
\end{aligned}
$$

The basal-friction law at iteration $k+1$ in the ice sheet is given by

$$
\left.\tau_{b, i+\frac{1}{2}}^{j, k+1} \equiv-C\left|u_{i+\frac{1}{2}}^{j, k}\right|^{\frac{1}{n}-1}\left[\frac{\left(\frac{\mathcal{N}_{i+1}^{j, k}+\mathcal{N}_{i}^{j, k}}{2}\right)^{n}}{\kappa\left|u_{i+\frac{1}{2}}^{j, k}\right|+\left(\frac{\mathcal{N}_{i+1}^{j, k}+\mathcal{N}_{i}}{2}\right.}\right)^{j, k}\right]^{\frac{1}{n}} u_{i+\frac{1}{2}}^{j, k+1},
$$

and similarly for the cell containing the grounding line. Basal stress in the ice shelf is zero for all iterations. Driving stress does not depend explicitly on $u$; it is computed entirely from time-independent quantities or thicknesses at iteration $k$. Boundary conditions are

$$
\begin{aligned}
& u_{\frac{3}{2}}^{j, k+1}=0, \\
& \frac{1}{\Delta x} v_{N+M}^{j, k}\left(u_{N+M+\frac{1}{2}}^{j, k+1}-u_{N+M-\frac{1}{2}}^{j, k+1}\right) \\
& \quad=\frac{1}{2} \rho_{\mathrm{i}}\left(1-\frac{\rho_{\mathrm{i}}}{\rho_{\mathrm{w}}}\right) g H_{N+M}^{j, k} .
\end{aligned}
$$

The result is a linear system involving a tridiagonal matrix

$$
\mathbf{M}_{u}^{k} \boldsymbol{u}^{k+1}=\boldsymbol{r}_{u}^{k} \text {. }
$$

We use a sparse matrix solver to compute the new velocities.

Then, we solve the continuity equation for the new thicknesses $H_{i}^{j, k+1}$ :

$$
\frac{H_{i}^{j, k+1}-H_{i}^{j-1}}{\Delta t}+\theta \mathcal{F}_{i}^{j, k+1}+(1-\theta) \mathcal{F}_{i}^{j-1}=a,
$$

where

$$
\mathcal{F}_{i}^{j, k+1} \equiv \frac{\left(H_{\mathrm{up}, i+\frac{1}{2}}^{j, k+1} u_{i+\frac{1}{2}}^{j, k+1}-H_{\mathrm{up}, i-\frac{1}{2}}^{j, k+1} u_{i-\frac{1}{2}}^{j, k+1}\right)}{\Delta x},
$$

and where

$$
H_{\mathrm{up}, i+\frac{1}{2}}^{j, k+1} \equiv\left\{\begin{array}{c}
H_{i}^{j, k+1} \text { if } u_{i+\frac{1}{2}}^{j, k+1} \geq 0, \\
H_{i+1}^{j, k+1} \text { if } u_{i+\frac{1}{2}}^{j, k+1} \leq 0 .
\end{array}\right.
$$

The boundary condition here is

$H_{2}^{j, k+1}-H_{1}^{j, k+1}=b_{2}-b_{1}$. 
The tridiagonal linear system (actually $u$ is typically strictly positive so the system is bidiagonal) is solved for the new thickness.

$\mathbf{M}_{H}^{k+1} \boldsymbol{H}^{k+1}=\boldsymbol{r}_{H}$.

Iteration continues until the residual

$R^{k} \equiv \mathbf{M}_{u}^{k} \boldsymbol{u}^{k}-\boldsymbol{r}_{u}^{k}<10^{-10} \max \left(\left|\tau_{d, i+\frac{1}{2}}^{j, k}\right|\right)$.

Note that all terms are evaluated at iteration $k$.

\section{C2 Addition of the GLP}

A few simple modifications to the numerical method from the previous section are required to add a grounding-line parameterization (GLP), as defined in Gladstone et al. (2010a). The basal and driving stresses in the grid cell containing the grounding line are modified so that the stresses transition smoothly between their grounded and floating values as the grounding line passes through the cell. Following Pattyn et al. (2006), we define the grounding-line position to be the location where the function $f_{\text {Pattyn }}(x)=H_{\mathrm{f}}(x) / H(x)$ is equal to one. Values of $f_{\text {Pattyn }}$ at arbitrary $x$ values are computed by linear interpolation between values at $H$-grid points:

$$
\begin{aligned}
f_{\text {Pattyn }}(x)= & \left(1-\frac{x-x_{i}}{\Delta x}\right) f_{i} \\
& +\left(\frac{x-x_{i}}{\Delta x}\right) f_{i+1} \quad x_{i} \leq x<x_{i+1},
\end{aligned}
$$

$f_{i} \equiv H_{\mathrm{f}}\left(x_{i}\right) / H_{i}$.

With this definition, the grounding-line position $x_{\mathrm{g}}$ is given by

$f_{\text {Pattyn }}\left(x_{\mathrm{g}}\right) \equiv 1$,

$x_{\mathrm{g}}=x_{i}+\frac{1-f_{i}}{f_{i+1}-f_{i}} \Delta x$.

Following Gladstone et al. (2010a), we define the fraction of the $u$-grid cell that is grounded as:

$\lambda_{g} \equiv \frac{1-f_{i}}{f_{i+1}-f_{i}}$.

Assuming the $u$-grid cell at $N+1 / 2$ contains the grounding line, the three stresses at the center of this cell are

$$
\begin{aligned}
T_{l, N+\frac{1}{2}} & =\frac{2 A^{-\frac{1}{n}}}{\Delta x^{1+\frac{1}{n}}}\left[H_{N+1}^{j}\left|u_{N+\frac{3}{2}}^{j}-u_{N+\frac{1}{2}}^{j}\right|^{\frac{1}{n}-1}\left(u_{N+\frac{3}{2}}^{j}-u_{N+\frac{1}{2}}^{j}\right)\right. \\
& \left.-H_{N}^{j}\left|u_{N+\frac{1}{2}}^{j}-u_{N-\frac{1}{2}}^{j}\right|^{\frac{1}{n}-1}\left(u_{N+\frac{1}{2}}^{j}-u_{N-\frac{1}{2}}^{j}\right)\right], \quad
\end{aligned}
$$

$$
\tau_{b, N+\frac{1}{2}}=-\lambda_{g} C\left|u_{N+\frac{1}{2}}^{j}\right|^{\frac{1}{n}-1} u_{N+\frac{1}{2}}^{j}\left[\frac{\left(\frac{\mathcal{N}_{N+1}^{j}+\mathcal{N}_{N}^{j}}{2}\right)^{n}}{\kappa\left|u_{N+\frac{1}{2}}^{j}\right|+\left(\frac{\mathcal{N}_{N+1}^{j}+\mathcal{N}_{N}^{j}}{2}\right)^{n}}\right]^{\frac{1}{n}},
$$

$$
\tau_{d, N+\frac{1}{2}}=-\rho_{\mathrm{i}} g \frac{\left(H_{N+1}^{j}+H_{N}^{j}\right)}{2}\left[\lambda_{g} \frac{\left(H_{N+1}^{j}-b_{N+1}-H_{N}^{j}+b_{N}\right)}{\Delta x}\right.
$$

$$
\left.+\left(1-\lambda_{g}\right) \delta \frac{\left(H_{N+1}^{j}-H_{N}^{j}\right)}{\Delta x}\right],
$$

where, as usual, the stress terms must balance:

$T_{l, N+\frac{1}{2}}+\tau_{b, N+\frac{1}{2}}+\tau_{d, N+\frac{1}{2}}=0$.

Note that $T_{l}$ has not been modified as part of the GLP because longitudinal-stress term takes the same form in the ice sheet and the ice shelf. The other equations of the system are unchanged from the previous section.

\section{C3 Chebyshev model}

The Chebyshev model is a Python code that finds steady-state solutions to the equations of motion. The code is pseudospectral (Boyd, 2001): integrals and derivatives are computed in spectral space (i.e., using Chebyshev modes), whereas products and quotients are computed in physical space on a collocation grid. The collocation points are the ChebyshevGauss-Lobatto nodes, defined as:

$x_{\mathrm{k}}=x_{\mathrm{g}} \frac{1-\cos \left(\frac{\pi k}{N}\right)}{2} \quad k=0,1, \ldots, N$,

where $N=1024$ is the order of the Chebyshev polynomials that form the set of basis functions. This choice of collocation grids means that there are collocation points on the domain boundaries - the ice divide $\left(x_{0}=0\right)$ and the grounding line $\left(x_{N}=x_{\mathrm{g}}\right)$ - which makes applying boundary conditions at these locations relatively straightforward. We used opensource code from PyBlog (von Winckel, 2013) for transformations between physical and spectral space and for recurrence relations for integrals and derivatives.

The code uses the derivative of thickness, $H_{x}$, as its primary computational variable. This choice reduces numerical noise because we never need to compute the second derivative of the primary variable. Higher-order derivatives introduce increasing amounts of numerical noise, whereas integrals tend to remove noise. The thickness $H$ is derived from $H_{x}$ by integration

$$
H=H\left(x_{\mathrm{g}}\right)+\int_{x_{\mathrm{g}}}^{x} H_{x^{\prime}} d x^{\prime},
$$


where $H\left(x_{\mathrm{g}}\right)$ is known from the flotation boundary condition, Eq. (9). The velocity $u$ is determined from $H$ using the integral of the steady-state continuity equation, $u H=a x$, and the velocity derivative $u_{x}$ is computed from the continuity equation, Eq. (1), with $H_{\mathrm{t}} \equiv 0$ :

$u=\frac{a x}{H}$

$u_{x}=\frac{a+u H_{x}}{H}$.

Given $u, H$ and their derivatives, we use Picard iteration to solve stress-balance, Eq. (2). Each Picard iteration involves solving the following linear system for $H_{x, k+1}$, based on the results computed at the previous iteration $k$ :

$$
\begin{aligned}
& \mathbf{M}_{k} \boldsymbol{H}_{x, k+1}=\boldsymbol{c}_{k}, \\
& M \equiv-\mathcal{D}\left(\boldsymbol{v}_{\mathrm{x}, \mathrm{k}} \boldsymbol{u}_{k}+\underline{v}_{k} \boldsymbol{u}_{\mathrm{x}, \mathrm{k}}+\boldsymbol{H}_{k}\right)-\mathcal{D}\left(\boldsymbol{v}_{k} \boldsymbol{u}_{k}\right) \cdot \mathbf{D}_{x}, \\
& \boldsymbol{c}_{k} \equiv-\boldsymbol{v}_{\mathrm{x}, \mathrm{k}} a-\boldsymbol{\tau}_{\mathrm{b}, \mathrm{k}}-\boldsymbol{H}_{k} \boldsymbol{b}_{\mathrm{x}, \mathrm{k}}, \\
& v \equiv 2 \bar{A}^{-\frac{1}{n}}\left|u_{x}\right|^{\frac{1}{n}-1},
\end{aligned}
$$

where quantities in bold italics are vectors of $N+1$ values from each collocation point, quantities that are bold and in capital letters are $(N+1) \times(N+1)$ matrices, $\mathcal{D}$ is an operator that puts the vector elements along the diagonal of a matrix, $\mathbf{D}_{x}$ is the Chebyshev derivative operator expressed as a matrix, and $\tau_{\mathrm{b}, \mathrm{k}}$ is given by Eq. (6) with all quantities evaluated at iteration $k$. Products of vectors are defined element-wise in these equations, rather than as dot products. Picard iteration requires an initial guess at $H_{x}$, which we define as

$H_{x, 0} \equiv 2\left[H\left(x_{\mathrm{g}}\right)-H_{0}\right] \frac{x^{2}}{x_{\mathrm{g}}^{2}}$,

$H_{0} \equiv 3000 \mathrm{~m}$.

An outer iterative loop uses Brent's root-finding method (Brent, 1973) to find the grounding-line position $x_{\mathrm{g}}$ where the final boundary condition, Eq. (13), is satisfied. Brent's method requires bounds on $x_{\mathrm{g}}$ : we break the domain into segments of downward-sloping bedrock topography and search for steady states independently on each segment. The method is also capable of finding unstable steady states on upwardsloping bedrock. The method is considered to have converged when both the residual $\boldsymbol{r}=\max \left(\left|\mathbf{M}_{k} \boldsymbol{H}_{\mathrm{x}, \mathrm{k}}-\boldsymbol{c}_{k}\right|\right)$ and the residual in Eq. (13) are less than $10^{-10}$. All computational variables are non-dimensionalized to be of order unity.
Acknowledgements. This work was supported by the Earth System Modeling and Scientific Discovery through Advanced Computing (SciDAC) programs funded by the US Department of Energy, Office of Science, Biological and Environmental Research and Advanced Scientific Computing Research. The Los Alamos National Laboratory is operated by the DOE National Nuclear Security Administration under Contract DE-AC52-06NA25396. The authors would like to thank Christian Schoof, Matt Hoffman, Steve Price and Ed Bueler for fruitful conversation. The authors also thank Rupert Gladstone, Frank Pattyn, an anonymous reviewer, and the editor, Olivier Gagliardini, for detailed comments that have improved the paper.

Edited by: O. Gagliardini

\section{References}

Boyd, J.: Chebyshev and Fourier Spectral Methods, Dover Books on Mathematics Series, Dover Publ., New York, 497-498, 2001.

Brent, R.: Algorithms for Minimization without Derivatives, Prentice-Hall, Englewood Cliffs, New Jersey, 47-52, 1973.

Budd, W. F. and Jenssen, D.: The dynamics of the Antarctic ice sheet, Ann. Glaciol., 12, 16-22, 1989.

Budd, W. F., Keage, P. L., and Blundy, N. A.: Empirical studies of ice sliding, J. Glaciol., 23, 157-170, 1979.

Bueler, E. and Brown, J.: Shallow shelf approximation as a "sliding law" in a thermomechanically coupled ice sheet model, J. Geophys. Res.-Earth, 114, F03008, doi:10.1029/2008JF001179, 2009.

Carter, S. P. and Fricker, H. A.: The supply of subglacial meltwater to the grounding line of the Siple Coast, West Antarctica, Ann. Glaciol., 53, 267-280, 2012.

Cornford, S. L., Martin, D. F., Graves, D. T., Ranken, D. F., Brocq, A. M. L., Gladstone, R. M., Payne, A. J., Ng, E. G., and Lipscomb, W. H.: Adaptive mesh, finite volume modeling of marine ice sheets, J. Comput. Phys., 232, 529-549, 2013.

Cuffey, K. and Paterson, W. S. B.: The Physics of Glaciers, Academic Press, p. 283, 2010.

Durand, G., Gagliardini, O., de Fleurian, B., Zwinger, T., and Le Meur, E.: Marine ice sheet dynamics: hysteresis and neutral equilibrium, J. Geophys. Res.-Earth, 114, F03009, doi:10.1029/2008JF001170, 2009.

Favier, L., Gagliardini, O., Durand, G., and Zwinger, T.: A threedimensional full Stokes model of the grounding line dynamics: effect of a pinning point beneath the ice shelf, The Cryosphere, 6, 101-112, doi:10.5194/tc-6-101-2012, 2012.

Fricker, H. A. and Scambos, T.: Connected subglacial lake activity on lower Mercer and Whillans Ice Streams, West Antarctica, 2003-2008, J. Glaciol., 55, 303-315, 2009.

Fricker, H. A., Coleman, R., Padman, L., Scambos, T. A., Bohlander, J., and Brunt, K. M.: Mapping the grounding zone of the Amery Ice Shelf, East Antarctica using InSAR, MODIS and ICESat, Antarct. Sci., 21, 515-532, 2009.

Gagliardini, O., Cohen, D., Raaback, P., and Zwinger, T.: Finite-element modeling of subglacial cavities and related friction law, J. Geophys. Res.-Earth, 112, F02027, doi:10.1029/2006JF000576, 2007. 
Gladstone, R. M., Payne, A. J., and Cornford, S. L.: Parameterising the grounding line in flow-line ice sheet models, The Cryosphere, 4, 605-619, doi:10.5194/tc-4-605-2010, 2010a.

Gladstone, R. M., Lee, V., Vieli, A., and Payne, A. J.: Grounding line migration in an adaptive mesh ice sheet model, J. Geophys. Res.-Earth, 115, F04014, doi:10.1029/2009JF001615, $2010 \mathrm{~b}$.

Gladstone, R. M., Payne, A. J., and Cornford, S. L.: Resolution requirements for grounding-line modelling: sensitivity to basal drag and ice-shelf buttressing, Ann. Glaciol., 53, 97-105, doi:10.3189/2012AoG60A148, 2012.

Goldberg, D., Holland, D. M., and Schoof, C.: Grounding line movement and ice shelf buttressing in marine ice sheets, J. Geophys. Res., 114, F04026, doi:10.1029/2008JF001227, 2009.

Hindmarsh, R. C. A.: A numerical comparison of approximations to the Stokes equations used in ice sheet and glacier modeling, J. Geophys. Res.-Earth, 109, 1-15, 2004.

Holland, P. R.: A model of tidally dominated ocean processes near ice shelf grounding lines, J. Geophys. Res.-Oceans, 113, C11002, doi:10.1029/2007JC004576, 2008.

Joughin, I. and Alley, R. B.: Stability of the West Antarctic ice sheet in a warming world, Nat. Geosci., 4, 506-513, 2011.

Larour, E., Schiermeier, J., Rignot, E., Seroussi, H., Morlighem, M., and Paden, J.: Sensitivity analysis of Pine Island Glacier ice flow using ISSM and DAKOTA, J. Geophys. Res.-Earth, 117, F02009, doi:10.1029/2011JF002146, 2012.

Le Brocq, A. M., Ross, N., Griggs, J. A., Bingham, R. G., Corr, H. F., Ferraccioli, F., Jenkins, A., Jordan, T. A., Payne, A. J., and Rippin, D. M.: Evidence from ice shelves for channelized meltwater flow beneath the Antarctic Ice Sheet, Nat. Geosci., 6, 945-948, 2013.

Lipscomb, W. H., Fyke, J. G., Vizcaino, M., Sacks, W. J., Wolfe, J., Vertenstein, M., Craig, A., Kluzek, E., and Lawrence, D. M.: Implementation and initial evaluation of the Glimmer Community Ice Sheet Model in the Community Earth System Model, J. Climate, 26, 7352-7371, doi:10.1175/JCLI-D-12-00557.1, 2013.

MacAyeal, D. R.: Large-scale ice flow over a viscous basal sediment: theory and application to ice stream B, Antarctica, J. Geophys. Res.-Sol. Ea., 94, 4071-4087, 1989.

Makinson, K., King, M. A., Nicholls, K. W., and Gudmundsson, G. H.: Diurnal and semidiurnal tide-induced lateral movement of Ronne Ice Shelf, Antarctica, Geophys. Res. Lett., 39, L10501, doi:10.1029/2012GL051636, 2012.

Martin, M. A., Winkelmann, R., Haseloff, M., Albrecht, T., Bueler, E., Khroulev, C., and Levermann, A.: The Potsdam Parallel Ice Sheet Model (PISM-PIK) - Part 2: Dynamic equilibrium simulation of the Antarctic ice sheet, The Cryosphere, 5, 727740, doi:10.5194/tc-5-727-2011, 2011.

Muszynski, I. and Birchfield, G. E.: A coupled marine ice-streamice-shelf model, J. Glaciol., 33, 3-15, 1987.

Pattyn, F.: A new three-dimensional higher-order thermomechanical ice sheet model: basic sensitivity, ice stream development, and ice flow across subglacial lakes, J. Geophys. Res.-Sol. Ea., 108, 2382, doi:10.1029/2002JB002329, 2003.

Pattyn, F., Huyghe, A., De Brabander, S., and De Smedt, B.: Role of transition zones in marine ice sheet dynamics, J. Geophys. Res.Earth, 111, F02004, doi:10.1029/2002JB002329, 2006.

Pattyn, F., Schoof, C., Perichon, L., Hindmarsh, R. C. A., Bueler, E., de Fleurian, B., Durand, G., Gagliardini, O., Gladstone, R., Goldberg, D., Gudmundsson, G. H., Huybrechts, P., Lee, V.,
Nick, F. M., Payne, A. J., Pollard, D., Rybak, O., Saito, F., and Vieli, A.: Results of the Marine Ice Sheet Model Intercomparison Project, MISMIP, The Cryosphere, 6, 573-588, doi:10.5194/tc6-573-2012, 2012.

Perego, M., Gunzburger, M., and Burkardt, J.: Parallel finiteelement implementation for higher-order ice-sheet models, J. Glaciol., 58, 76-88, 2012.

Pimentel, S., Flowers, G. E., and Schoof, C. G.: A hydrologically coupled higher-order flow-band model of ice dynamics with a Coulomb friction sliding law, J. Geophys. Res.-Earth, 115, F04023, doi:10.1029/2002JB002329, 2010.

Pollard, D. and DeConto, R. M.: Description of a hybrid ice sheetshelf model, and application to Antarctica, Geosci. Model Dev., 5, 1273-1295, doi:10.5194/gmd-5-1273-2012, 2012.

Rignot, E., Velicogna, I., Van den Broeke, M. R., Monaghan, A., and Lenaerts, J.: Acceleration of the contribution of the Greenland and Antarctic ice sheets to sea level rise, Geophys. Res. Lett., 38, L05503, doi:10.1029/2011GL046583, 2011.

Rutt, I. C., Hagdorn, M., Hulton, N. R. J., and Payne, A. J.: The Glimmer community ice sheet model, J. Geophys. Res.-Earth, 114, F02004, doi:10.1029/2011GL046583, 2009.

Schoof, C.: The effect of cavitation on glacier sliding, Proc. R. Soc. A, 461, 609-627, doi:10.1098/rspa.2004.1350, 2005.

Schoof, C.: Ice sheet grounding line dynamics: steady states, stability, and hysteresis, J. Geophys. Res, 112, F03S28, doi:10.1029/2006JF000664, 2007a.

Schoof, C.: Marine ice-sheet dynamics - Part 1: The case of rapid sliding, J. Fluid Mech., 573, 27-55, 2007b.

Schoof, C. and Hindmarsh, R. C.: Thin-film flows with wall slip: an asymptotic analysis of higher order glacier flow models, Q. J Mech. Appl. Math., 63, 73-114, 2010.

Tulaczyk, S., Kamb, W. B., and Engelhardt, H. F.: Basal mechanics of Ice Stream B, West Antarctica: 1. Till mechanics, J. Geophys. Res.-Sol. Earth, 105, 463-481, 2000a.

Tulaczyk, S., Kamb, W. B., and Engelhardt, H. F.: Basal mechanics of Ice Stream B, West Antarctica: 2. Undrained plastic bed model, J. Geophys. Res.-Sol. Earth, 105, 483-494, 2000 b.

van der Wel, N., Christoffersen, P., and Bougamont, M.: The influence of subglacial hydrology on the flow of Kamb Ice Stream, West Antarctica, J. Geophys. Res.-Earth, 118, 97-110, doi:10.1029/2012JF002570, 2013.

Vaughan, D. G. and Spouge, J. R.: Risk estimation of collapse of the West Antarctic ice sheet, Clim. Change, 52, 65-91, 2002.

Velicogna, I.: Increasing rates of ice mass loss from the Greenland and Antarctic ice sheets revealed by GRACE, Geophys. Res. Lett., 36, L19503, doi:10.1029/2009GL040222, 2009.

Vieli, A. and Payne, A. J.: Application of control methods for modelling the flow of Pine Island Glacier, West Antarctica, Ann. Glaciol., 36, 197-204, 2003.

Vieli, A. and Payne, A. J.: Assessing the ability of numerical ice sheet models to simulate grounding line migration, J. Geophys. Res.-Earth, 110, F01003, doi:10.1029/2004JF000202, 2005.

von Winckel, G.: PyBlog, available at: http://www.scientificpython. net/pyblog.html (last access: 30 September 2013), 2013.

Weertman, J.: Stability of the junction of an ice sheet and an ice shelf, J. Glaciol., 13, 3-11, 1974.

Whillans, I. M. and van der Veen, C. J.: The role of lateral drag in the dynamics of Ice Stream B, Antarctica, J. Glaciol., 43, 231237, 1997. 
Wingham, D. J., Siegert, M. J., Shepherd, A., and Muir, A. S.:

Rapid discharge connects Antarctic subglacial lakes, Nature, 440, 1033-1036, 2006. 